\title{
L'« enquête artistique » : les méthodes de représentation artistique $d u$ monde du travail en question
}

The artistic inquiry: questionning the artistic representation methods of the working worlds

Jan Middelbos

\section{OpenEdition}

Journals

Édition électronique

URL : https://journals.openedition.org/itti/1197

DOI : 10.4000/itti. 1197

Éditeur

Université de Poitiers

Référence électronique

Jan Middelbos, «L'« enquête artistique » : les méthodes de représentation artistique du monde du travail en question », Images du travail, travail des images [En ligne], 2 | 2016, mis en ligne le 01

septembre 2016, consulté le 25 juillet 2021. URL : http://journals.openedition.org/itti/1197 ; DOI : https://doi.org/10.4000/itti. 1197

Ce document a été généré automatiquement le 25 juillet 2021.

Images du travail, travail des images 


\title{
L'« enquête artistique » : les méthodes de représentation artistique du monde du travail en question
}

\author{
The artistic inquiry: questionning the artistic representation methods of the \\ working worlds
}

Jan Middelbos

1 Sans doute, la question des méthodes de représentation ${ }^{1}$ des mondes du travail a-t-elle pu traverser nombre de discussions proposées par les ateliers d'«Image du travail, Travail des images » (février 2013). Et pour cause, ce colloque, intitulé plus précisément Image du travail, travail des images. Pratiques artistiques, démarches scientifiques, s'était donné comme objectif « d'échanger et de confronter les points de vue entre, d'une part, les chercheurs en sciences sociales spécialisés dans le travail et mobilisant l'image dans ce cadre, et d'autre part, les professionnels de l'image, fixe ou animée, engagés dans une démarche documentaire dans ce champ. [Il s'agissait] de comparer les méthodes et les types de connaissances proposés par ces deux démarches, d'appréhender leurs points communs et leurs différences, de mesurer leurs apports et influences réciproques. ${ }^{2} "$

2 Cependant, si nous supposons également une proximité des méthodes déployées à la fois par les « chercheurs en sciences sociales » et les " professionnels de l'image », nous souhaitons la considérer plus particulièrement eu égard à l'importance que ces deux démarches pourraient accorder au «terrain» et à leurs habitants. En effet, lorsque l'artiste cherche à produire des images documentaires du monde du travail il est confronté aux mêmes questions méthodologiques que le chercheur en sciences sociales (sociologue ou ethnographe) placé en situation de prélever des données sur son terrain d'enquête. De cette façon, la représentation documentaire, comme l'enquête sociologique sur et dans le monde du travail, suppose pour ces professionnels un enchaînement partagé de questions méthodologiques pratiques (comment accéder à 
son terrain, quelles données prélever, comment les prélever et avec quels médias d'enregistrement, comment monter et quelle forme donner à ces matériaux recueillis, dans quel lieu les restituer et pour quel public, etc. ?) mais aussi éthiques et politiques (comment gagner la confiance de nos interlocuteurs, quelle place donner à la subjectivité des acteurs dans le travail de représentation, comment palier aux déséquilibres subjectifs entre représentants et représentés, comment trahir - ou au contraire ne pas trahir - l'hospitalité de ceux qui nous accueillent, etc. ?).

ous utilisons le terme d'enquete artistique dans le titre de cet article, c'est qu'il fait directement référence à l'artiste placé en situation de représentation des mondes du travail car, comme le sociologue placé en situation d'enquête sur son terrain, l'artiste aura à faire appel à l'enregistrement du "réel » - pour secréter en quelque sorte ses propres documents, ses propres sources - et sera comme lui exposé aux mêmes questions et travers méthodologiques. Cependant, et étant donné que l'usage même du terme d'enquête artistique peut porter à confusion, nous tenons à préciser ici que ce terme ne désigne pas une enquête sociologique qui serait menée par des sociologues sur le monde de l'art et des artistes. De même, étant donné que le propre de l'art - s'il y a - est bien d'échapper à toute définition des rôles impartis, il ne s'agit pas ici de dire que la fonction de l'artiste est de produire des enquêtes, mais plutôt de dire que lorsqu'il s'agira pour lui de s'introduire dans l'entreprise afin de rendre visible dans l'espace public ce qui est habituellement caché derrière les portes closes des univers privés de production, l'artiste se placera alors dans une situation et activera des méthodes comparables à celles expérimentées par le chercheur en sciences sociales placé en situation d'enquête de terrain. Dans ces deux positions, cela implique, d'une façon pragmatique, de s'introduire sur un terrain afin d'en prélever des données (le moment de l'enregistrement ethnographique ou documentaire), de les agencer dans un certain ordre (le moment du montage réflexif) puis de les restituer en d'autres mondes (le moment de la recontextualisation par le choix du contexte de monstration, du support et de l'adresse au public). Ce qui fait qu'en parlant de méthodes d'enquête sociologique nous ne cesserons de parler de méthodes de représentation artistique et vice versa.

4 Ainsi, s'il est important de comprendre les difficultés rencontrées par les sociologues ou les artistes - dans leurs approches du terrain, il est aussi utile d'interroger les façons de monter et de restituer les données qui y sont prélevées pour comprendre d'où ils parlent - c'est-à-dire à partir de quelle position distincte - afin de saisir l'engagement qui sous-tend leur démarche.

Investir un terrain d'enquête implique pour le sociologue - comme pour l'artiste - de s'ingérer dans un contexte qui lui est la plupart du temps étranger. Et on peut constater que les représentations réalisées par les artistes dans l'entreprise relèvent le plus souvent - en termes sociologiques - d'une observation plutôt que d'une observation participante. De la même façon, les méthodes d'approche de l'artiste vers l'entreprise pour en faire l'enregistrement documentaire - sont principalement de deux ordres :

1. L'artiste prélève ses données à partir d'une position extérieure à l'entreprise : l'artiste déclaré ou non déclaré comme tel - doit négocier - ou faire négocier - son « entrée dans la place » afin d'obtenir de la direction son autorisation et bénéficier ainsi de l'hospitalité de ses hôtes.

2. L'artiste prélève ses données à partir d'une position intérieure à l'entreprise : l'artiste déclaré ou non déclaré comme tel - se fond dans l'entreprise, il est - ou feint d'être ${ }^{3}$ - l'un de ses membres à part entière. 
6 Les artistes ne représenteront donc pas les mondes du travail de la même façon selon qu'ils sont invités par l'entreprise à le faire ou qu'ils sont salariés de l'entreprise ; selon qu'ils cherchent à s'y introduire ou qu'ils l'habitent déjà. Leurs rapports méthodologiques sont différents et leurs points de vue se distinguent en fonction de la place et du statut qu'ils occupent dans l'entreprise. Il est donc utile de distinguer la représentation artistique menée de l'extérieur par l'artiste-invité de la représentation artistique participative menée de l'intérieur par le salarié-artiste (Middelbos, 2011-2012, 11-12).

7 L'artiste-invité, autorisé par la direction à entrer sur le lieu de production (et en tant que nouvel entrant), se place vis-à-vis des salariés dans une position suspecte quant à la fonction particulière qui lui revient dans l'entreprise et aux connivences qu'il peut entretenir avec la direction qui l'a introduit. Lorsque l'artiste-invité est intronisé sur le lieu du travail, il peut apparaître, aux yeux des travailleurs qui lui servent de modèle, comme un étranger dont ils ne savent ni d'où il vient ni où il va. De plus, l'artiste-invité se trouve exposé à reproduire les divisions du travail traditionnelles : d'un côté l'artiste représente, et de l'autre, son modèle est représenté.

8 Selon qu'ils se situent d'un côté ou de l'autre de la médiation, représentants et représentés n'y trouvent pas les mêmes intérêts. Si le modèle peut bien accepter librement d'être représenté (quand bien même sa position de salarié ne lui permet pas forcément de refuser), il subordonne sa subjectivité artistique au profit de celle de l'artiste, qui en conserve le monopole exclusif. De cette façon, la prééminence de la subjectivité de l'auteur crée de facto un effacement de la subjectivité de son modèle (les travailleurs). La division du travail est intégrée et naturellement reproduite par l'artiste lorsque celui-ci accepte de représenter vis-à-vis de son modèle (le travailleur isolé) son unité et sa volonté artistique, ou comme le disent Karl Marx et Friedrich Engels dans L'idéologie allemande $(1976,397)$ : «La concentration exclusive du talent artistique chez quelques individualités, et corrélativement son étouffement dans la grande masse des gens, est une conséquence de la division du travail. ${ }^{4}$ " La représentation des mondes du travail, qui résulte d'une "puissance étrangère " (l'artiste comme auteur professionnel de la représentation), n'apparaît pas aux travailleurs comme leur propre puissance conjuguée, basée sur une coopération volontaire des deux parties (Marx, 1969, 266-267) ${ }^{5}$. L'engagement de l'artiste vis-à-vis de ses modèles - et de leur hospitalité - varie en fonction de la place qu'il occupe et de la place qu'il laisse occuper aux modèles, à toutes les étapes de la représentation (de l'élaboration à l'exposition des résultats). À défaut, les pratiques de représentation révèleront toujours des conditions et des intérêts antagonistes et placeront les sujets dans des positions asymétriques qui pourront faire apparaitre un déséquilibre subjectif entre les professionnels de la représentation et les travailleurs représentés.

9 Aussi, l'ingérence de l'artiste - comme celle du sociologue - dans les mondes du travail se réalise souvent dans un partage inégalitaire des savoirs et des pouvoirs (en particulier lorsque les représentants occupent dans l'espace social une position supérieure à celle de leurs modèles représentés). Ce partage inégalitaire peut être la conséquence de la prédominance et de la survalorisation de la qualité d'auteur, mais aussi celle $\mathrm{du}$ caractère particulièrement décontextualisant de la restitution des documents produits, et cela qu'elle ait lieu dans le cadre performatif de l'art ou dans celui de publications (artistiques comme scientifiques). 
10 En effet, la réception par le public-lecteur de tout document prélevé - ou enquête effectuée - dans les mondes du travail est influencée par son contexte de présentation. Ainsi, là où le document s'expose comme catégorie esthétique (art documentaire), le document (qu'il relève d'une image fixe ou animée) devient œuvre et sa fonction documentaire n'est plus sa seule fonction. Si la monstration se produit par exemple dans le cadre d'institutions artistiques, la perception du document s'en trouve modifiée. Le document pourra être ainsi réévalué selon des critères esthétiques dans son ensemble, et analysé tant dans son processus de construction que dans sa formalisation et sa restitution.

11 Cet article se propose donc de revenir sur ces interrogations en discutant plus particulièrement des méthodes activées par les professionnels de l'image documentaire pour produire des représentations des travailleurs et des mondes du travail qu'ils habitent. Et si le volet méthodologique peut sembler secondaire par rapport aux multiples entrées que le document produit peut avoir à offrir, il nous semble au contraire que les méthodologies déployées par les artistes peuvent nous aider à comprendre la généalogie de ces déséquilibres subjectifs entre l'auteur et son modèle et venir lever le voile sur des pratiques de négociation qui ont cours, la plupart du temps hors champ de l'image, entre professionnels de la représentation d'un côté et travailleurs représentés de l'autre. En ce sens, nous chercherons à dévoiler ce hors champ de la cuisine interne propre à certains professionnels de la représentation en analysant leurs productions à l'aune des conditions de leur fabrication. Nous chercherons à appréhender l'acte de représentation lui-même et concentrerons notre attention sur les moyens et manières de faire mis en œuvre pour y parvenir. À l'aide d'un corpus artistique placé sous la catégorie esthétique de l'art documentaire, nous voulons comprendre la dialectique instaurée entre fabricants de représentations (qu'ils soient photographes, cinéastes, artistes,...) et travailleurs pour saisir comment ces acteurs reproduisent ou, au contraire, tentent d'échapper aux divisions du travail entre représentants et représentés. Ainsi, avant de nous demander quelle position le document défend par rapport à l'invisibilité des travailleurs, nous voulons nous demander quelle est leur place dans ces mêmes rapports de production. Cette question vise la place et la fonction qui revient à l'auteur - mais aussi au travailleur, son modèle - comme producteur - ou coproducteur - d'œuvres.

12 Si nous souhaitons également faire retour sur cette question posée en guise de préambule à ce colloque de février 2013, c'est qu'il nous semble que les grands absents à la confrontation de ces points de vues - entre "chercheurs en sciences sociales spécialisés dans le travail » d'un côté et "professionnels de l'image engagés dans une démarche documentaire dans ce champ » de l'autre - sont les travailleurs eux-mêmes. Bien que présents et invités à participer à ce colloque (et l'on peut noter la présence remarquable - entre autres - de l'ouvrier-écrivain Hubert Truxler, alias Marcel Durand, (Durand, 2006), de l'ouvrière-écrivaine Sylviane Rosière (Rosière, 2010), de l'ancien ouvrier et syndicaliste Christian Corouge (Corouge et Pialloux, 2011) ou encore de l'agent d'entretien-artiste Aurélie Le Faurestier, le point de vue singulier de ces travailleurs - qui ont fait des formes d'auto-représentation au travail le moyen même de témoigner des mondes qu'ils habitent - ne peut trouver sa place dans ce débat méthodologique entre "chercheurs en sciences sociales" et "professionnels de l'image ». La position et la fonction singulières de ces derniers pourraient bien masquer malgré eux les pratiques de ces travailleurs - qu'ils soient écrivains et/ou artistes - qui 
entendent donner, de l'intérieur même des mondes du travail, un autre point de vue, à la fois sur des conditions de travail et d'exploitation expérimentées mais aussi sur les conditions bien particulières d'en rendre compte.

13 C'est pourquoi, il s'agira bien d'affirmer ici qu'il existe au travers de la figure du salarié-artiste une culture et une histoire parallèle des pratiques de représentation qui proviennent des travailleurs eux-mêmes et qui, par des moyens d'expression divers (trans-médiatiques), cherchent à en donner un point de vue de l'intérieur mettant ainsi en question l'unique point de vue des professionnels de la représentation (quelles que soient les disciplines à partir desquelles ils se situent) et leurs manières de faire.

Il s'agira donc de savoir comment l'artiste-invité, qui cherche à pallier à l'invisibilité des travailleurs, pourrait-il rétablir méthodologiquement ces déséquilibres subjectifs produits de la division du travail entre représentants et représentés, mais aussi quel autre point de vue le salarié-artiste peut-il proposer de l'intérieur même des mondes du travail qu'il habite au quotidien.

\section{Une représentation non négociée : les travailleurs mis en scène}

Pour illustrer notre propos, nous voulons nous arrêter tout d'abord sur une recherche que nous avons réalisée en 2010 sur le travail photographique de Nicolas Faure qui nous semble emblématique d'une conception de la représentation, que certains mondes de l'art se font et entendent donner des mondes du travail, mais aussi emblématique de certaines formes actuelles de résidences d'artiste en entreprise proposées par certaines institutions artistiques.

Pour cela, nous avons cherché à comprendre comment le photographe a négocié ses représentations avec ses modèles, les travailleurs. Cette série photographique, intitulée Portraits/Chantiers (Faure, Lacoue-Labarthe et Nancy, 2004), réalisée en 1999 est le résultat d'un travail que Nicolas Faure a effectué pour une commande de la ville de Strasbourg. Cette commande visait l'accompagnement artistique de la ligne B du tramway qui s'implantait alors à Strasbourg. L'initiative de ce projet revient à Michel Krieger ${ }^{7}$ qui préside et nomme le comité d'expert chargé - entre autres - de sélectionner les projets artistiques. Et c'est Christian Bernard ${ }^{8}$ qui sera désigné comme «chef de projet artistique » pour la Ligne $\mathrm{B}^{9}$ du tramway ${ }^{10}$.

Pour répondre au cahier des charges, les artistes ${ }^{11}$ devaient proposer des projets artistiques qui répondent à un certain besoin de communication autour de la construction de la ligne B du tramway de la ville. C'est au travers de cet événement l'intégration d'un tramway accompagnée d'un projet artistique dans l'environnement urbain de la ville - que le volontarisme politique s'exprime et s'affiche. La mairie (PS) entend accroître la notoriété de sa ville, sur un plan à la fois local et international. Et cela même si le délégué à la commande publique Michel Krieger défend ses choix artistiques, en disant avoir " voulu privilégier la cohérence, l'insertion dans le site, le dialogue avec la population ${ }^{12}$ ", et en reléguant la notoriété de certains artistes à un "plus» et "en aucun cas [à] un but ${ }^{13}$ ", il reste que l'on est bien ici, face à une opération promotionnelle de la ville, dont les artistes se retrouvent être les artisans sous-traitants, travaillant tout à la fois aux bénéfices de leur réputation et à la réussite économique de leur propre entreprise prestataire de service. En répondant à l'appel 
d'offre, les artistes s'emploient à la tâche dans le cadre spécifique d'un contrat nonsalarié et se distinguent ainsi de la condition ouvrière salariale tant par les rémunérations perçues que par la nature même des tâches quotidiennes prescrites et imparties. Les artistes prestataires de services sont ainsi convoités pour leur capacité à produire du «lien social» et leur rayonnement symbolique international est tout particulièrement apprécié ${ }^{14}$.

Même si, pour ce comité d'expert, la notoriété n'est pas le seul effet recherché, les artistes sous-traitants ont répondu à une commande publique, liée à un contexte bien particulier: celui d'un plan de communication artistique qui accompagne l'inauguration des lignes du tramway. Et c'est dans ce contexte que le pouvoir politique a passé commande à Nicolas Faure ${ }^{15}$. Concernant les honoraires de l'artiste prestataire de service, nous n'avons pas d'autres informations que ce qu'a bien voulu en dire le conseiller municipal Harry Lapp (opposition UDF) au conseil municipal du 8 octobre 1999:

«Mais franchement, concernant la galerie des portraits, payer un photographe 130000 Frs pour photographier des ouvriers, je ne sais pas si celui-ci leur donne des cachets comme aux mannequins, mais les pauvres, s'ils apprennent que le photographe va gagner 130000 Frs pour faire des clichés, sans le tirage et tout ce qui suit bien évidemment, ils vont s'interroger ${ }^{16}$. "

C'est donc dans ces conditions spécifiques de contrat à la teneur idéologique entrepreneurial que Nicolas Faure s'est retrouvé en position de photographier les ouvriers de ce chantier. L'artiste-invité ne peut alors que s'éloigner de ses modèles (les ouvriers de chantier) dont il entend faire le portait.

Photographie 1. Stéphane Fond, 1999.

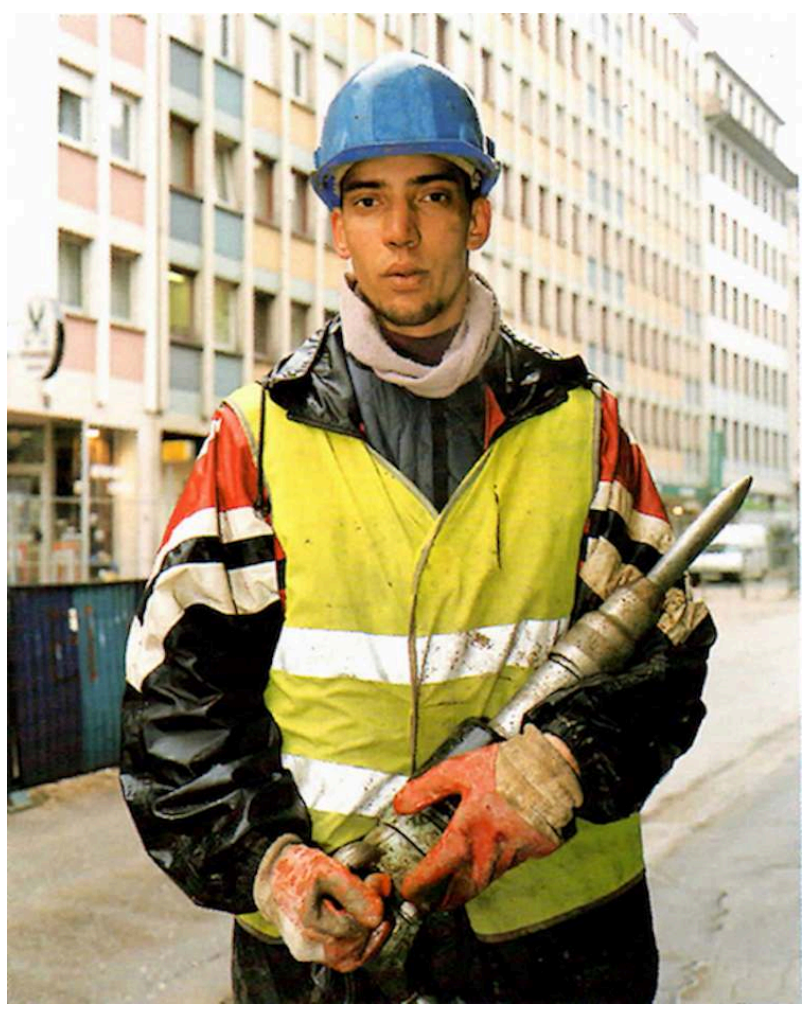

Nicolas Faure. 
Le philosophe Philippe Lacoue-Labarthe, qui a écrit un texte intitulé «Eu Égard » pour le catalogue de Nicolas Faure, dit au sujet de ces photographies: «Voici donc ceux qu'on ne regarde jamais; et qui le savent" (Lacoue-Labarthe, 2004, 11). On peut pourtant se demander quel acte photographique peut bien recouvrir ce «Voici »? Et quelle méthodologie le photographe a-t-il pu mobiliser pour rendre visibles «ceux qu'on ne regarde jamais »?

Donner à voir « ceux qu'on ne regarde jamais » ou rendre visible les invisibles signifiait pour lui photographier et exposer ces travailleurs en grand format sur les panneaux publicitaires de la ville de Strasbourg, y compris sur le trajet et pendant la mise en service du tramway (durant une période de deux semaines). Cette série photographique de Nicolas Faure représente des ouvriers qui se retrouvent formellement centrés dans l'image, en couleurs, la plupart du temps en portrait américain et, de temps en temps, en pied. Il a également fait inscrire - en gros, en gras et en guise de légende sur l'affiche - le nom et prénom de chaque employé représenté. Le photographe - et la municipalité par sa commande - s'étaient donc fixés pour objectif de donner de la visibilité aux ouvriers de ce chantier, qui, malgré leurs gilets fluorescents, restent invisibles aux yeux du plus grand nombre. Il s'agissait d'une forme de récompense "symbolique », et non d'un remerciement par le salaire (Lacoue-Labarthe, 2004, 32) ${ }^{17}$.

Ce projet sert donc - sans engager un apport financier plus important dans les salaires ou dans l'amélioration des conditions de travail - une forme de reconnaissance "symbolique», mais aussi «instrumentale» par la récompense qu'elle permet de délivrer. Ce besoin de reconnaissance, auquel fait appel le photographe pour attirer des modèles devant l'appareil, est confirmé par ce qu'en dit Stéphane Fond ${ }^{18}$ qui a accepté de poser pour le photographe et de répondre à mes questions :

«C'était un gros chantier. J'étais jeune, j'avais 20 ans, j'étais fier de poser [pour le photographe $]^{19}$."

23 Ce besoin de reconnaissance semble amplifié par la dureté des conditions de travail :

«Les conditions étaient dures. Il fallait déblayer $20 \mathrm{~m}^{3}$ à l'heure. C'est là que j'ai connu la fraternité entre collègues. La sécurité n'était pas bien assurée. On a eu un blessé grave. Il s'est brûlé avec une flamme due au gaz. Moi je me suis électrocuté à plusieurs reprises. Quand la commission venait, ça allait, mais dès qu'elle était repartie, c'était dur ${ }^{20}$.»

Le problème c'est qu'aucun partage des situations de travail vécues par les salariés ne semble avoir interrompu le photographe dans son travail de représentation. Et le processus de «négociation » entamé par l'artiste - son " offre de parole » - est somme toute très minimale: les ouvriers n'ont fait que choisir les outils avec lesquels ils auraient tenus à être associés. La systématicité du processus de mise en scène photographique nous permet aussi de douter des modes de "négociation » engagés avec eux : sur les photographies, les ouvriers posent tous avec « leurs [supposés] outils de prédilection », regardent fixement l'appareil, et portent quasiment tous une chasuble fluo. Il est difficile d'envisager que la production de ces images s'est réalisée en dehors des seules intentions de l'artiste. De plus, la négociation engagée par Nicolas Faure avec les ouvriers de ce chantier est forcément affaiblie par la position de l'artiste. Le photographe-prestataire, qui répond à un appel d'offre, travaille d'abord, comme on l'a vu, pour ses commanditaires. On peut dire que ces derniers accompagnent le photographe partout où il se déplace, et qu'ils sont indissociablement liés à sa démarche lorsque ce nouveau venu intervient sur le chantier. Pour les ouvriers du 
chantier, le photographe est à la fois le représentant de la municipalité - en quelque sorte mandaté par elle (au sens de la Vertretung ${ }^{21}$ - et détenteur exclusif des formes de représentation qu'il va pouvoir fabriquer à partir de ses modèles, les travailleurs.

Cependant, s'il s'agit bien d'une forme de reconnaissance qui vient d'en haut et qui entend prendre à témoin l'ensemble des habitants de la ville, Bruno Charneau (situé au centre de la photographie) n'a pour sa part jamais su ce que Nicolas Faure allait faire de ces photographies. C'est moi qui le lui ai appris par téléphone. Et comme tous les salariés avec lesquels je me suis entretenu, il n'a pas été invité à voir son portrait exposé lors du vernissage, résultat de sa représentation. Pour les ouvriers du chantier, la reconnaissance de leur travail s'est donc limitée à l'acte de prise de vue.

Ainsi, les travailleurs de ce chantier sont désappropriés de leur image et ne décident pas des espaces et conditions dans lesquels ils sont photographiés et exposés. Ils n'ont aucun contrôle sur le format de leur image, sur la légende et les commentaires qui l'accompagnent, sur leur participation à telle exposition plutôt qu'à telle autre,... Malgré les intentions annoncées par l'artiste, les ouvriers restent silencieux sur leurs propres expériences. Ils sont "parlés ", d'une parole experte - celle du photographe, des philosophes, des commissaires d'exposition, des critiques d'art,... - et jamais ne parlent en leur propre nom, de manière à ce qu'ils puissent devenir maitres de la formulation de leurs propres besoins ou désirs (Bourcier, 2001, 141).

Les photographies des ouvriers en grand format placardées dans la ville sur des panneaux publicitaires nous montrent les ouvriers de manière à les remarquer, mais elles ne les représentent que comme cliché. Montrer un ouvrier avec son outil à la main et en tenue de chantier, c'est en donner une représentation stéréotypée, réduite à une image codifiée. L'individu photographié ne l'est que comme représentant symbolique d'une catégorie : les ouvriers de chantier. Plutôt que de rendre compte de la singularité des personnes et/ou des conditions de leur activité, leurs représentations semblent ici mettre en scène bien plus une condition abstraite, un statut social fantasmé qui effacent alors toute particularité des sujets et toute particularité de leur environnement de travail. La subjectivité de l'artiste et son esthétique associée imposent ainsi un déséquilibre dans les relations qu'il entretient avec les sujets qu'il entend représenter. Le but de l'artiste dans ces conditions d'approche n'est plus tant de représenter les travailleurs, que de réaliser « une image selon certaines conventions formelles, le référent concret de l'image photographique compte moins qu'une référence globale à la fois au monde du travail et aux manières plastiques consacrées de l'appréhender.» (Rouillé, 1984, 32) En effet, nous pouvons constater que l'artiste, représentant professionnel, «sacrifie l'effet de réel à l'effet esthétique, c'est-à-dire organise sa mise en scène moins à partir d'une situation réelle que d'images préexistantes. " (Rouillé, 1984, 34) Si les travailleurs sont les éléments principaux de la représentation, ils n'en sont pas toujours la raison. 
Photographie 2. Franco Martin, Bruno Charneau, Sébastien Muths, 1999.

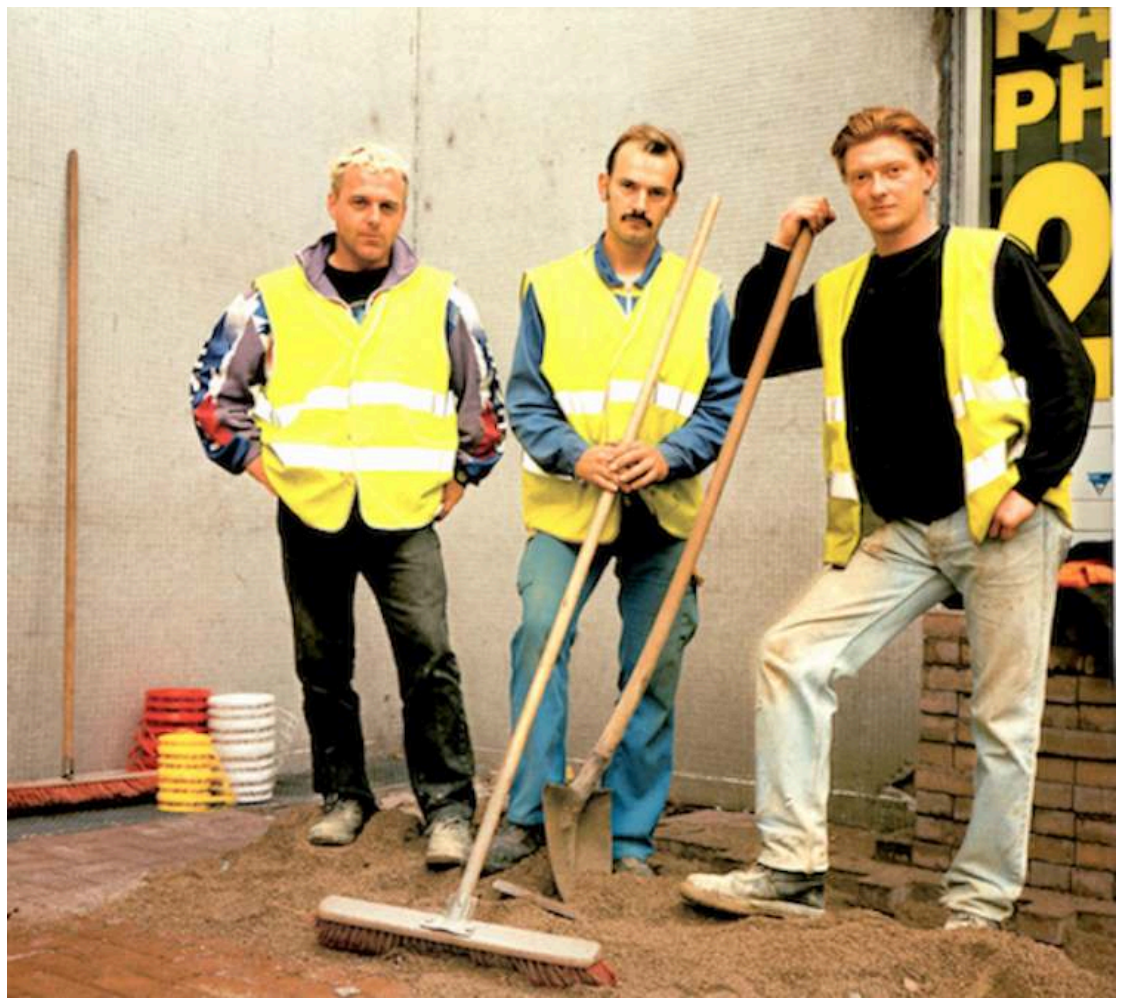

Nicolas Faure.

Même si certains salariés ont refusé de jouer les figurants ${ }^{22}$ (sans doute la seule possibilité réellement laissée aux salariés d'interagir sur le processus), il n'est pas facile de se soustraire à ce genre d'exercice, comme il n'est pas toujours facile de refuser de se plier à une tâche prescrite et exigée dans le cadre d'un travail salarié. En l'occurrence, pour ce qui est de la photographie de Stéphane Fond, c'est son chef de chantier qui lui a proposé de poser pour le photographe; et Bruno Charneau (au centre de la photographie) et Sébastien Muths (à droite) posent tous deux avec leur chef direct Franco Martin (à gauche). Comment le photographe peut-il savoir, dans cette situation, s'il a en face de lui un salarié qui répond à une énième tâche demandée par son employeur ou un sujet libre de modifier un processus décidé en dehors de lui ?

Le hors champ, constitué par le contrat qui lie le représenté à son représentant, existe bel et bien, même s'il n'est que rarement revendiqué et assumé comme faisant partie intégrante de l'œuvre par les professionnels de la représentation. Cependant, si les conditions de représentation sont, dans le travail de Nicolas Faure, reléguées hors champ de l'image nous voulons évoquer ici le travail de Philip-Lorca diCorcia qui, dans sa série photographique intitulée Hollywood (également connu sous le nom The Hustlers), annonce de suite dans ses légendes une partie des conditions qu'il a négociées avec ses modèles, pour que ceux-ci acceptent de poser pour lui. 
Photographie 3. Marilyn, 28 years old, Las Vegas, Nevada, \$30, 1990-92.

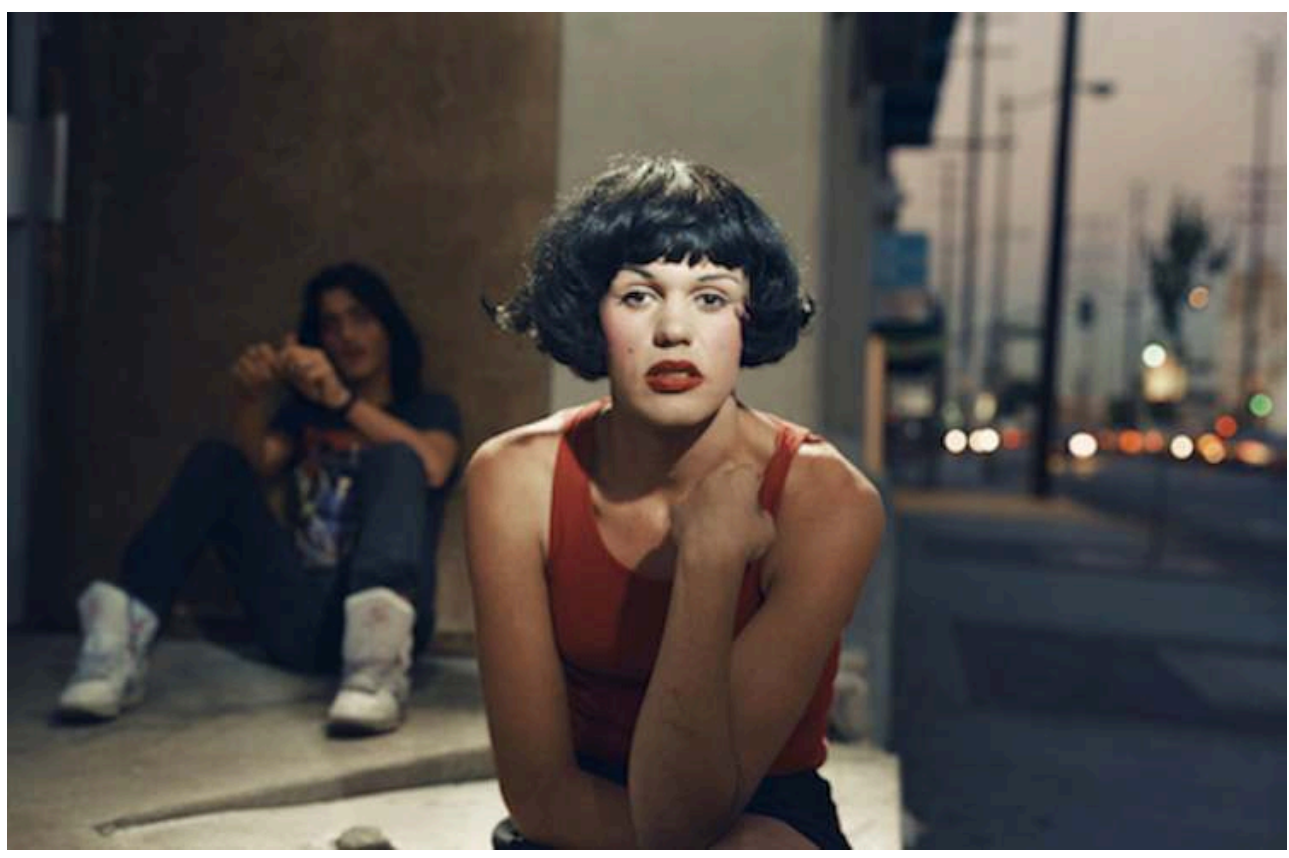

Philip-Lorca diCorcia.

Photographie 4. Mike Miller, 24 years, Allentown, Pennsylvania, \$25, 1990-92.

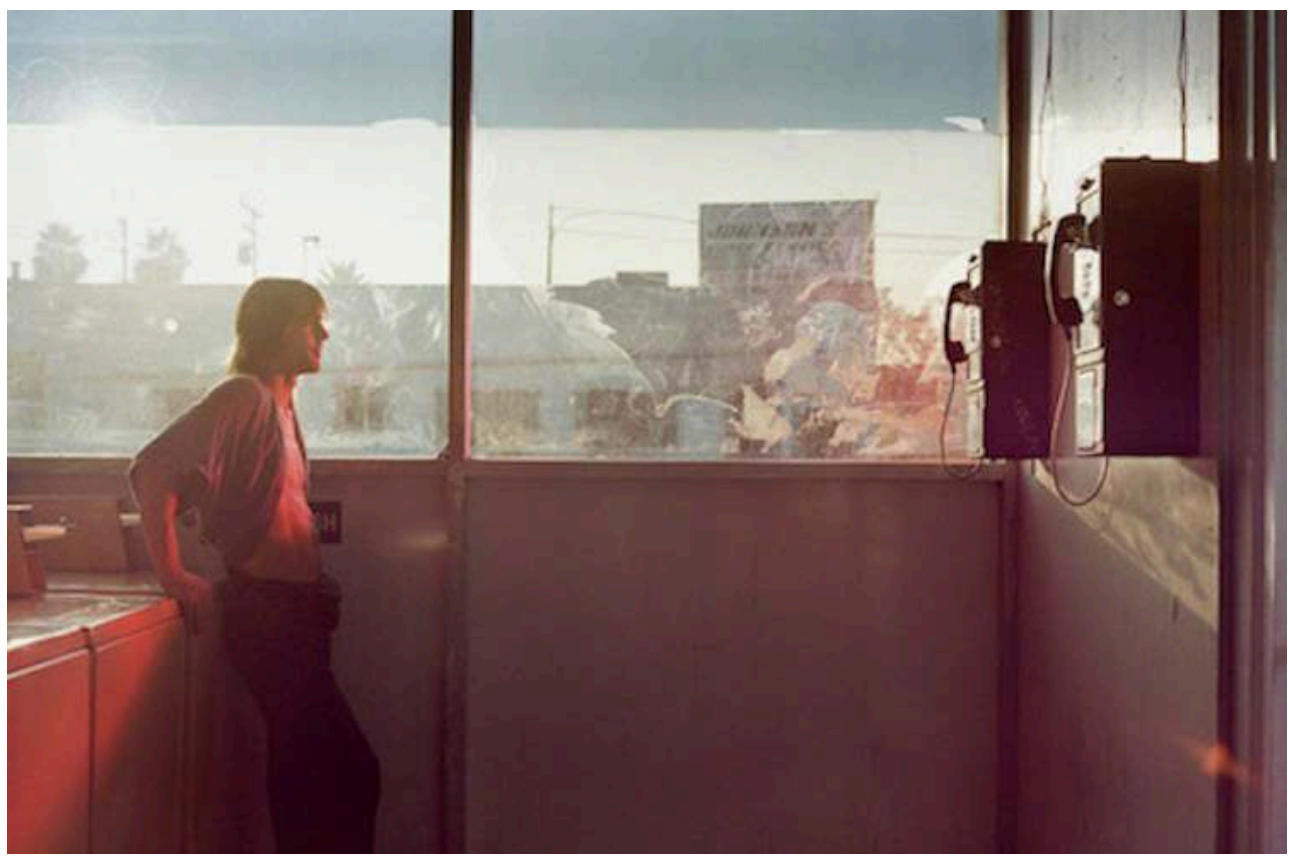

Philip-Lorca diCorcia.

30 Les modèles du photographe sont ici des travailleurs du sexe qui se prostituent sur les boulevards. Il les met en scène dans des chambres d'hôtels ou directement dans la rue sur le boulevard. Le processus de négociation que le photographe a entamé avec eux est simple. Il paye son modèle le prix d'une passe. Puis il indique, dans ses légendes photographiques, le prénom et le nom du modèle (ou son pseudonyme), son âge, la ville et l'État d'où sont originaires les modèles, la somme d'argent que le photographe a 
négocié avec son modèle, et la date de la série. L'artiste Philip-Lorca diCorcia assume ainsi de rompre avec ce tabou d'un contrat habituellement négocié hors champ, en intégrant dans le champ de la représentation le pacte qui le lie à ses modèles.

Et si cette méthode - négocier un tarif avec son modèle - peut sembler particulièrement cynique et brutale, son approche marchande des sujets qu'il veut représenter dans leur contexte de travail - sur les boulevards ou dans les chambres d'hôtels - nous apparaît ici a minima assumée et revendiquée. Il paye pour un service, pour mettre en scène un modèle et la brutalité des rapports marchands qui ont cours dans la prostitution est respectée. L'artiste ne triche pas, la légende est indissociablement liée à l'acte photographique et à son processus de négociation. Par cette légende, il permet au spectateur-lecteur de réaliser une mise à distance critique du processus même de représentation, d'une représentation négociée. Cette recherche de la bonne distance par rapport au modèle - assumée ici comme insaisissable dans sa totalité - est à rapprocher de la « distance éthique ${ }^{23}$ » que Raymond Depardon cherche à opérer entre lui et ses modèles représentés, bien que d'une façon formellement différente, puisque sa recherche est celle d'une « bonne distance » physique - optique.

Cependant, si Jean-Claude Moineau dit du photographe diCorcia qu'il «procure au moins aux jeunes hommes qu'il photographie, montés à Hollywood dans l'espoir de "faire du cinéma" et qui, pour toute réussite, sont tombés dans la prostitution, un court moment, sous les sunlights, de la gloire qu'ils étaient venus chercher » (Moineau, 2008, 147), ceux-ci n'en sont pas moins dépossédés des manières de recontextualiser ces photographies, pourtant objets résultants de leur représentation. Là encore les modèles sont dépossédés des manières de recontextualiser ces photographies (elles le seront dans le cadre performatif - et marchand - de l'art : galeries, musées, livres d'art, etc.), autrement dit les divisions du travail entre représentant et représentés perdurent et ne sont en rien modifiées par la procédure mise en place par l'artiste. De cette façon, « division du travail et propriété privée sont des expressions identiques, la première exprimant par rapport à l'activité ce que la seconde exprime par rapport au produit de cette activité. » (Marx et Engels, 1976, 47) Le produit (la photographie) qui est l'objet résultant de la division du travail entre représentant et représentés, demeure l'unique propriété du photographe. 
Photographie 5. Vue de l'exposition de Philip-Lorca diCorcia intitulée Hustlers, David Zwirner Galley, du 12 Septembre 2012 au 2 novembre 2013, New York.

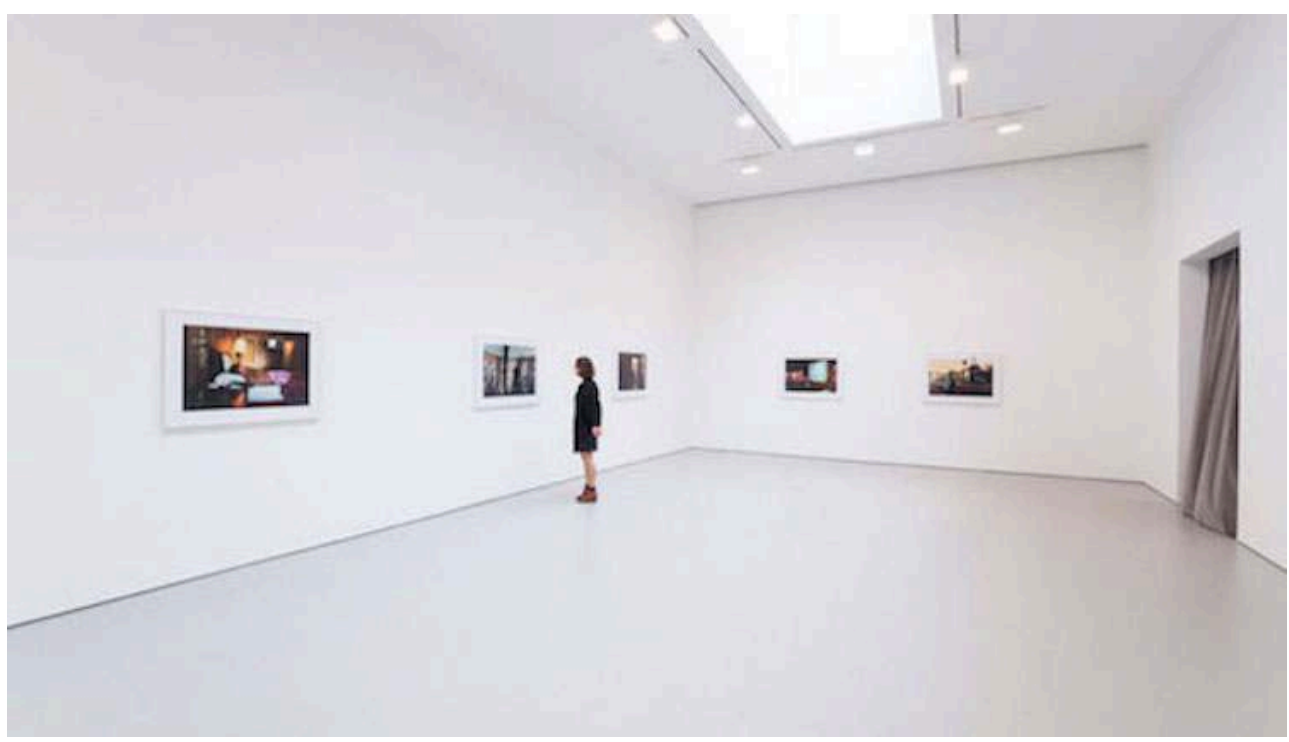

Photographie 6. Vue de l'exposition de Philip-Lorca diCorcia intitulée Hustlers, David Zwirner Galley, du 12 Septembre 2012 au 2 novembre 2013, New York.

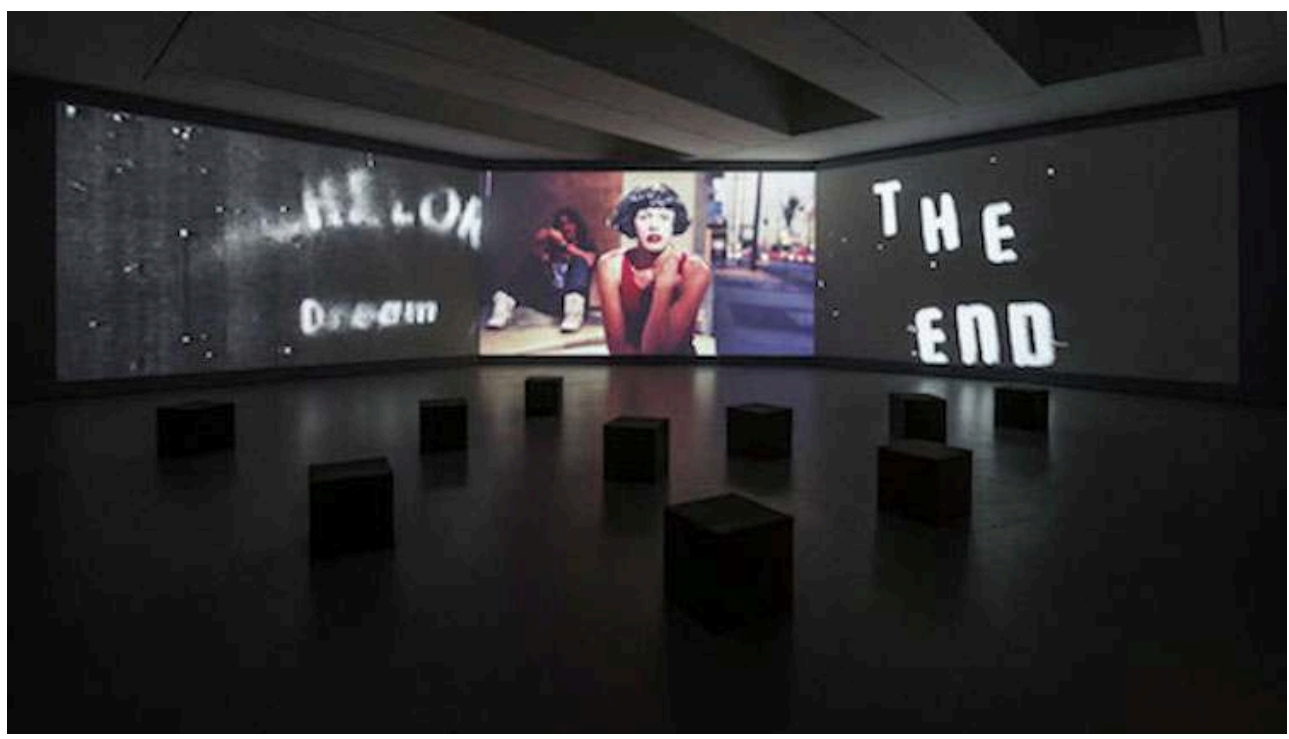

Mais, associer des sujets subalternes, en proie à la précarité et à la violence de la rue, aux modalités même de la représentation ne va pas de soi, comme en témoigne la réponse que l'artiste a fait à la question posée par Lynne Tillman :

" - [Les modèles] ont-ils contribué à choisir leurs scènes?

- À peu près $80 \%$ d'entre eux non rien voulu faire d'autre, à part demander immédiatement leur argent et essayé de partir, parce qu'ils sont des prostitués. » (Simpson, 2007, 95)

C'est bien la nature du contrat, qui lie l'exposé - le travailleur - à son exposant l'artiste -, qui pose question. Le contrat d'échange tacite, proposé par le représentant à son représenté - comme celui proposé par l'enquêteur à son enquêté -, nous semble être de même nature que la transaction qu'un employeur peut opérer avec son salarié telle que l'explicite le sociologue Nicolas-Le Strat: 
«Au moment de la transaction, [le salarié] est institué partenaire de l'échange, libre de disposer et de contracter. Mais, dès l'accord conclu, il s'engage à obéir, se place sous l'autorité de l'employeur. En fait il s'accepte comme employé, c'est-à-dire consent à exercer son travail sous la subordination (au sens juridique) de quelqu'un. Il efface alors sa volonté et exécute son activité sous le contrôle d'un employeur. Dualité et paradoxe, car pour participer à la division sociale du travail, il doit prendre figure de sujet libre, tandis que pour occuper sa fonction dans l'ordre de la production, il ne peut être que subordonné. » (Nicolas-Le Strat, 1989)

Subordonnés à la seule subjectivité de l'artiste, les sujets représentés apparaissent exclus des façons même d'être représentés. On est bien ici face à ce que le sociologue appelle une "logique de la dissociation », qui conduit à autonomiser les champs et les domaines de compétences de chacun.

En effet, représenter les salariés sur leur lieu et pendant leur temps de travail, c'est les aborder dans des situations bien particulières. Les travailleurs, n'étant toujours pas émancipés du règne de la nécessité, se salarient et contractualisent contre un revenu leur capacité à exercer une activité physique et/ou intellectuelle. Et en marchandant contre un salaire leur force de travail, ils renoncent donc à jouir de leur image, à jouir de leur identité particulière pour entrer dans des formes de représentation salariale (Nicolas-Le Strat, 1989).

37 En reproduisant les divisions du travail, c'est-à-dire en excluant les sujets producteurs (travailleurs), des modalités même de leur représentation (conditions de monstration comprises) et en acceptant ainsi d'en être les seuls maîtres de conception, les artistes ne se comportent-ils pas, in fine, comme des Directeurs des ressources humaines (DRH) au service de leur propre entreprise?

\section{Coopération entre représentant et représentés : vers une auto-représentation des travailleurs}

Cependant, il est des exceptions qui aspirent à faire exploser ces catégories entre représentant d'un coté et représenté de l'autre. Soit en instaurant des méthodes de coopération volontaire (artistes et travailleurs travaillant à un même objectif), soit par des formes d'«auto-représentation » au travail. Pour ce qui est des coopérations volontaires, comme nous le verrons au travers du film La Charnière des groupes Medvedkine, cela implique de la part des représentants (des cinéastes comme professionnels de la représentation) un certain renoncement à leur subjectivité d'auteur (ou une mise au service de leur subjectivité d'auteur) au nom d'un processus collectif de création, et de la part des représentés (des travailleurs) qu'ils aient la volonté - et la possibilité - de se réapproprier les outils de représentation pour leur propre compte. Autant dire tout de suite que ces moments sont difficiles à négocier et si rares qu'ils ne semblent pas avoir fait école (ni en sociologie ni en art). C'est sans doute pourquoi, dans cette volonté d'échapper aux catégories (entre représentant et représenté), on manque moins d'exemples d'auto-représentation au travail, qui, au travers d'enquêtes sociologiques mais aussi artistiques, témoignent directement des conditions d'existence vécues au travail. Et si nous convoquons les groupes Medvedkine aujourd'hui, c'est qu'ils constituent encore un précédent méthodologique novateur et sans commune mesure pour ce qui est de la représentation documentaire des mondes du travail et des travailleurs qui l'habitent. 
39 Ainsi, la situation de l'artiste-invité se complique lorsque les modèles (les travailleurs) se « rebiffent " pour reprendre l'expression de Xavier Molénat ${ }^{24}$. Le film La Charnière (Bonfanti et Cèbe, 1968) du groupe Médvedkine de Besançon rend très bien compte de ces moments où les travailleurs expriment leur mécontentement quant à la manière dont ils ont été représentés. Ce film sans images est extrait de la bande-son d'un débat d'avril 68 qui a suivi la projection du film À bientôt j'espère... réalisé par Chris Marker et Mario Marret. À bientôt j'espère... relatait la grève menée en 1967 par les salariés de la Rhodiacéta à Besançon (branche textile du groupe Rhône-Poulenc). Projeté aux anciens grévistes de la Rhodia, il fut vigoureusement remis en cause par une partie de ce public qui ne s'y reconnaissait pas. La Charnière s'ouvre sur l'intervention d'un travailleur en colère :

«Je pense que si les travailleurs de la Rhodia ont vu ça et que si c'est ce que le réalisateur a vraiment voulu exprimer, alors vraiment c'est un incapable. Si vraiment il a voulu exprimer ce que ressentent les travailleurs de Rhodia et le besoin qu'ils ont, je pense que le réalisateur est un incapable. Je le dis crûment. Je le dis tel que je le pense, mais je pense plutôt vrai. Je le dis crûment aussi, que là y a simplement une exploitation des travailleurs de Rhodia, par des gens qui, parait-il, luttent contre le capitalisme. » (Bonfanti et Cèbe, 1968)

Ce média ne peut être affiché ici. Veuillez vous reporter à l'édition en ligne http:// journals.openedition.org/itti/1197

Si tous les intervenants ne sont pas aussi radicaux, beaucoup ne s'y retrouvent pas et relèvent les manques et les failles de ce film. Cependant, Chris Marker, conscient des « limites» et des «éléments d'échecs » de son film, invitera les ouvriers à porter eux même à l'écran leur propre vision des mondes qu'ils habitent et les encouragera - d'un ton un peu paternaliste - à passer pratiquement derrière la caméra pour s'autoreprésenter en formulant cette hypothèse :

« Nous [cinéastes professionnels], on sera toujours au mieux des explorateurs bien intentionnés, plus ou moins sympathiques mais de l'extérieur, et que de même que pour sa libération, la représentation et l'expression du cinéma de la classe ouvrière sera son œuvre à elle-même. Et c'est quand les ouvriers auront entre leurs mains les appareils audiovisuels, qui nous montreront à nous les films sur la classe ouvrière et sur ce que c'est qu'une grève, et sur ce que c'est que l'intérieur de l'usine. Mais je crois qu'on serait même dix mille fois plus malin et moins romantique, qu'on serait quand même limité par cette espèce de réalité cinématographique qu'on expérimente tout le temps, qu'on aille chez les ouvriers ou chez les pingouins, que évidemment on ne peut exprimer réellement que ce qu'on vit. » (Bonfanti et Cèbe, 1968)

Est-ce à dire que nous ne pourrions exprimer réellement que ce qu'on vit ? Il ne s'agit pas de dire que Classe de lutte - premier film réalisé après À bientôt j'espère... et La Charnière par le groupe de Besançon constitué d'ouvriers et de techniciens du cinéma est « en soi » meilleur ou moins bon qu'À bientôt j'espère... - réalisé uniquement par des professionnels -, ou encore de dire que «les sujets opprimés parlent, agissent et savent pour eux-mêmes, [ce qui conduirait effectivement] à une politique essentialiste et utopique. » (Spivak, 2009, 27) Mais plutôt de dire qu'il apparait méthodologiquement plus juste, si l'on veut tenter d'échapper aux divisions du travail entre représentants et représentés, de construire de part en part une œuvre collectivement assumée et de considérer la question de savoir "qui montre? " au même titre que celle de savoir « quoi montrer?» 

renverser les rôles et les tâches assignés à chacun dans l'acte de représentation et cherchent ainsi une coopération volontaire des salariés autour d'objectifs politiques et artistiques définis en commun. Mais faire en sorte que les représentés deviennent acteurs de leur propre représentation n'est pas simple. Le seul volontarisme des artistes ne suffit pas toujours à convaincre et à construire de part en part une œuvre collectivement assumée. En dépit de ces tentatives d'inverser les rôles attribués à chacun, les divisions du travail persisteront dans la fabrication des films à venir. Les habitudes l'emportent et les professionnels de la représentation se verront assignés par les ouvriers de reprendre en charge les outils cinématographiques (Muel, 2000, 29-30)25. Mais si ces négociations sont aussi difficiles c'est que la coréalisation de films documentaires demande aux ouvriers du temps et réclame d'épouser un double statut, celui d'ouvrier-cinéaste. Faire des films exige d'être ouvrier le jour et cinéaste la nuit et les week-ends, pour reprendre le titre du film Week-end à Sochaux (Muel, ibid., 27-29) ${ }^{26}$. Toutefois, malgré ces difficultés, ce négoce annonçait les débuts fructueux d'une coopération au travers entre autres du film Classe de lutte, premier film réalisé après $\grave{A}$ bientôt j'espère... et La Charnière par le groupe de Besançon constitué d'ouvriers-cinéastes et de cinéastes-militants.

, cette volonté des travailleurs de la Rhodia de donner une autre version du travail s'était déjà exprimée en 1967 par la formulation d'une nouvelle revendication dans les protocoles de fin de grève. Bruno Muel (cinéaste-militant dans les groupes Medvedkine et participant comme invité au colloque «Images du travail, travail des images » de février 2013) rend compte des propos tenus par des travailleurs de la Rhodia qui exigeaient de leur patron un droit à l' "auto-représentation " à l'usine : «lors des négociations devant aboutir à la reprise du travail, les grévistes présentèrent aux patrons une revendication inédite qui les étonna beaucoup. Ils demandaient le droit de filmer eux-mêmes, ou de faire filmer par un de leurs amis cinéastes, leurs postes de travail. Ils disaient : vous prenez le droit de réaliser des films industriels techniques ou de promotion en nous filmant sans nous demander notre avis. Vous donnez une image de notre travail qui est la vôtre, quoi de plus normal que nous puissions présenter notre version qui est différente. Bien entendu les patrons refuseront cette demande en se réfugiant derrière le secret industriel. » (Muel, ibid., 18). Par cette simple demande, les travailleurs de la Rhodia révélaient un déficit démocratique au sein du monde du travail et démontraient que la représentation de son univers était subordonnée aux décisions de la direction, elles-mêmes protégées par le régime de la propriété privée des moyens de production ${ }^{27}$. Si désormais les téléphones portables se sont substitués aux caméras Beaulieu et permettent leur introduction plus facilement sur les lieux de travail, leurs usages ne se passent pas pour autant d'un certain savoir-faire.

\section{Auto-représentation artistique au travail et détournement tactique}

Une autre figure, qui offre de nombreuses similarités avec celle de l'ouvrier-cinéaste est celle du salarié-artiste. Le salarié-artiste fait du lieu de travail un territoire propre à l'exercice quotidien de sa pratique artistique. À la différence de l'artiste-invité, le salarié-artiste - comme l'ouvrier-cinéaste - a intégré l'espace de travail par nécessité «économique ». Quand bien même ses pratiques se réclament du nom d'art, le salarié-

Images du travail, travail des images, 2 | 2016 
artiste n'est pas placé sous la protection d'un statut d'artiste autorisé et reconnu par la direction de l'entreprise (comme ça peut être le cas des résidences d'artistes en entreprise ${ }^{28}$ ). Il fait de sa condition salariale et des contraintes quotidiennes qui y sont associées la matière première de sa pratique artistique. Contrairement à des observateurs extérieurs, il peut parler à partir de la place qui est la sienne et se prendre comme son propre modèle. Par cet effort d'auto-représentation, qui lui permet d'objectiver sa position dans le monde du travail, il accroît sa vigilance quant aux contraintes qui s'exercent sur lui. Accéder à la possibilité de s'auto-représenter au travail devient donc un enjeu à la fois pour l'artiste mais aussi pour le travailleur qui cherche à donner une autre version du travail. L'auto-représentation au travail lui permet, par exemple, au travers "d'enquêtes artistiques participatives ", de témoigner directement des conditions d'existence vécues au travail.

Il en va ainsi de l'attitude du peintre Laurent Marissal qui, employé (d'avril 1997 à janvier 2002) comme agent de surveillance au Musée Gustave Moreau, utilisait «à des fins picturales le temps aliéné à la Direction des Musées de France. » (Marissal, 2005, 41) L'artiste use de nombreux subterfuges pour déjouer le regard menaçant des caméras de vidéo surveillance. Il fait de chacun d'eux des œuvres. Avec Sieste clandestine, il réalise un autoportrait vidéo sur son lieu et pendant son temps de travail ${ }^{29}$. Il se dessine deux yeux ouverts qu'il colle sur ses paupières pour faire une sieste. Cette autoreprésentation au travail nous donne à voir l'artiste en train de se « coltiner » le réel et de faire un apprentissage tactique de son contexte de travail. Il prend à contre-pied des injonctions autoritaires jusqu'à l'absurde en les déjouant avec ironie. L'artiste-gardien de salle répond ainsi aux stratégies menées par l'encadrement du musée par des formes de détournement et de résistance tactique. Mais Laurent Marissal étend aussi le domaine de son œuvre à des résistances sociales moins discrètes. Après avoir mis en place avec ses collègues une "première plate-forme de revendications" (Texte signé par les agents le 14 septembre 1997), il crée une active section syndicale (déclarée le 6 janvier 1998 sous le nom: section CGT-Gustave Moreau) à des fins picturales, pour «modifier réellement l'espace et le temps au musée.» (Marissal, 2005, 97) Pour ce qui est de la métamorphose de l'espace, ce sont les treizième et quatorzième points (sur quarante) de cette plate-forme de revendications qui les portent. On peut lire dans le chapitre "amélioration des conditions d'hygiène et de sécurité »: 13. Installer un vestiaire et une salle de repos pour les agents dignes de ce nom (pièce trop petite pour 6 personnes). 14. Installer des toilettes séparées pour le personnel et le public, avec distinction homme/femme. Et c'est sous la contrainte syndicale menée par l'artiste que ces points de revendication aboutiront à un redimensionnement physique des espaces. La Direction des musées de France (DMF) se voyant dans l'obligation de programmer des travaux pour aménager - entre autres - « une salle de pause, des vestiaires, de doter le musée de nouveaux locaux pour l'administration (un appartement de fonction est acheté par la DMF) et d'aménager des réserves. Des réunions avec l'architecte [sont également organisées pour permettre] aux agents d'exprimer leur désir... Lors de ces réunions, [Laurent Marissal ne pourra s'empêcher] de faire modifier la répartition des espaces et [fera] déplacer une cloison modifiant le volume des espaces. " (Marissal, 2005, 150) 
Document 1. Plans.

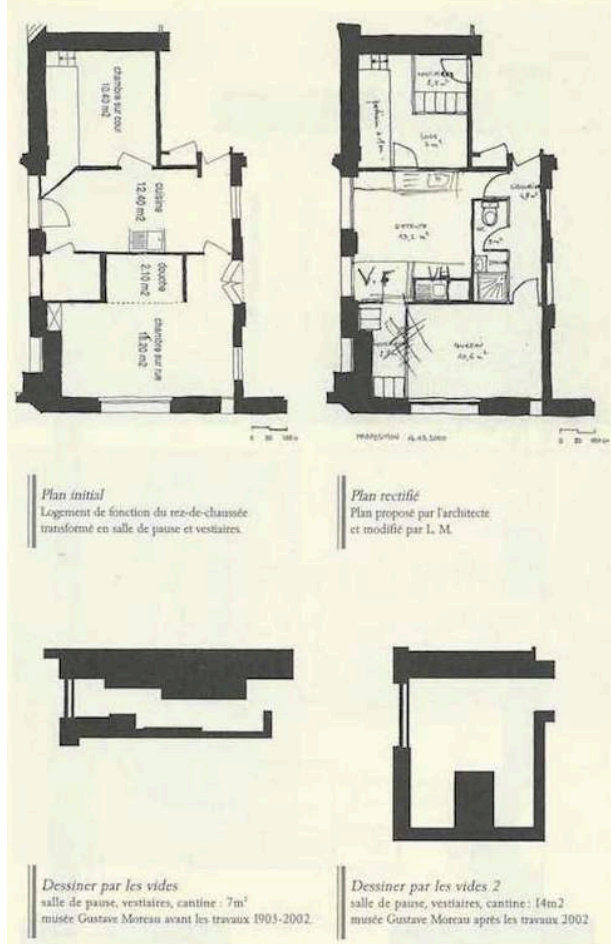

Laurent Marissal (2005), Pinxit, Rennes, Incertain Sens, p 151.

Laurent Marissal. 
Document 2. Plan définitif.

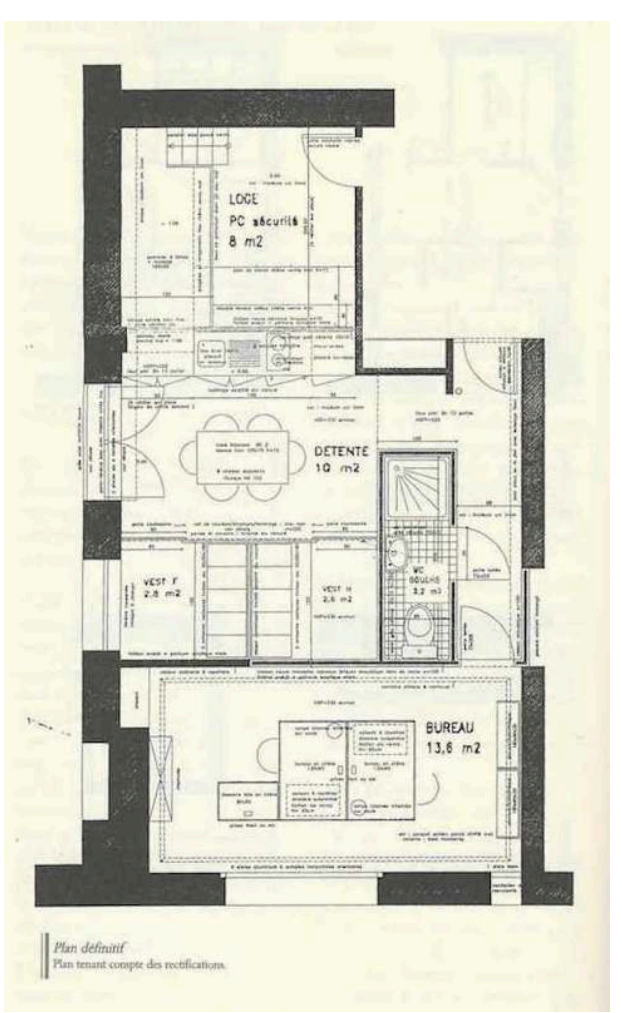

Laurent Marissal (2005), Pinxit, Rennes, Incertain Sens, p 152.

Laurent Marissal. 
Document 3. Ruines/chantier.
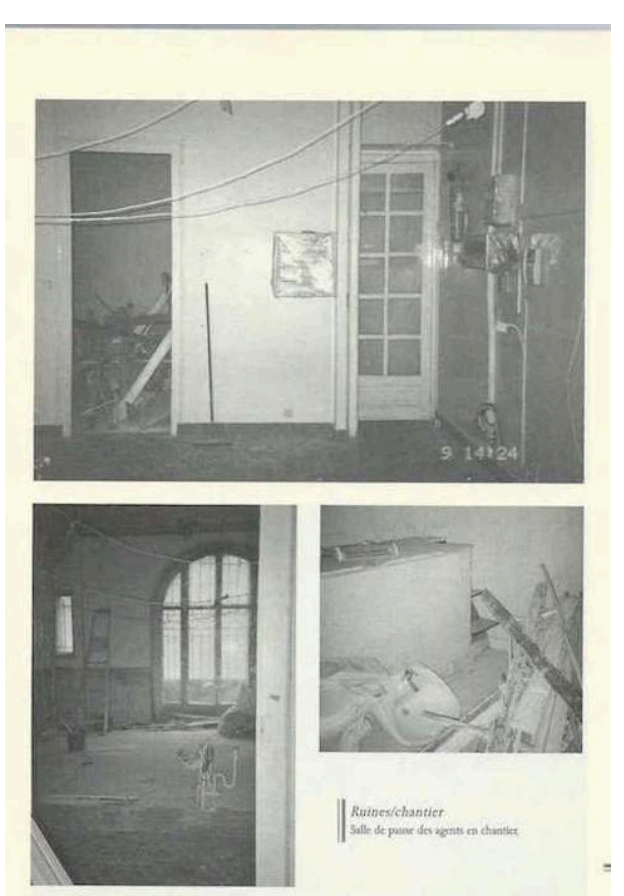

Laurent Marissal (2005), Pinxit, Rennes, Incertain Sens, p 153.

Laurent Marissal. 
Document 4. Intérieur.
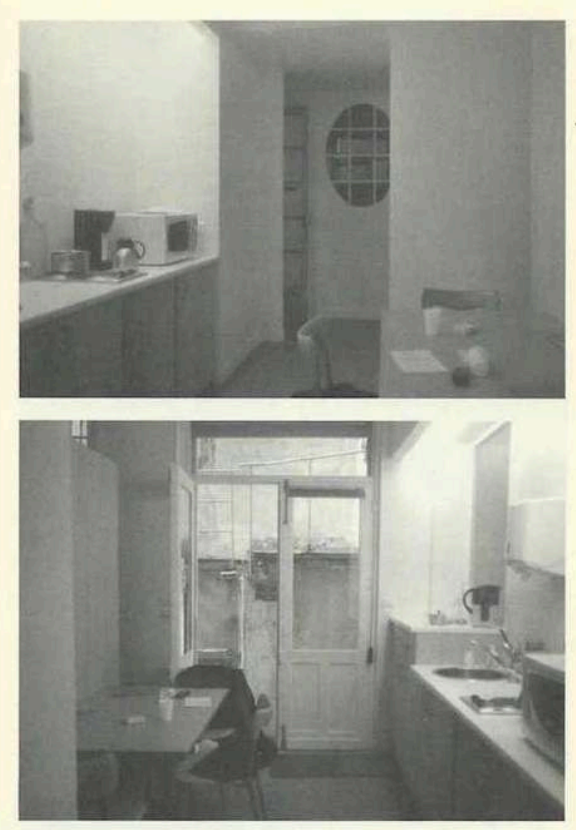

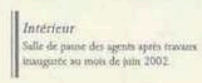

Laurent Marissal (2005), Pinxit, Rennes, Incertain Sens, p 155.

Laurent Marissal.

47 Par ces différentes actions, Laurent Marissal tente, à partir de sa position de salariéartiste, un renversement des rapports de forces qui s'exercent sur lui. Il se sert de son lieu de travail comme d'un territoire de jeu pour mettre en place des formes de détournement qui lui appartiennent : «À son insu, le musée rémunère une production dont il n'aura pas la jouissance.» (Marissal, 2005, 1) Mais Laurent Marissal ne se contente pas d'actions performatives. Il observe, enquête et se fait aussi collectionneur en documentant systématiquement les données qui le concernent tout en tenant le registre méthodique de ses agissements. Par des positionnements et repositionnements tactiques (par exemple, sortir la caméra au bon moment et la placer au bon endroit), il cherche à rendre visible les détournements qu'il opère sur l'univers privé (ou semiprivé) de son contexte de travail. Aussi, sa pratique de représentation et d'autoreprésentation au travail permet à l'artiste de faire état de la diversité de ses « actions picturales » au Musée Gustave Moreau dans le bilan qu'il dresse à la fin de son livre Pinxit (a peint). 
BILAN

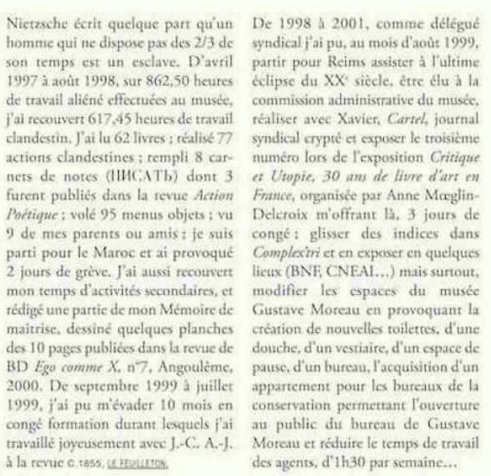

\section{Décrire son terrain d'enquête c'est représenter, représenter c'est « trahir »}

Représenter le monde du travail, à partir d'une position de sociologue ou d'artiste, relève de procédures méthodologiques qui peuvent se révéler étroitement liées et à de nombreux égards comparables. Quand bien même, à la différence des artistes qui peuvent prendre cette liberté de modifier le contexte sur lequel ils interviennent, les sociologues - cherchant souvent de façon illusoire à neutraliser la situation d'enquête (Mauger, 1991, 128) - sont tenus sur leurs terrains par un souci de rigueur scientifique, il n'en reste pas moins qu'un compte-rendu d'enquête de terrain peut être perçu comme un travail documentaire, une "photographie sociologique». Avec Walter Benjamin et par le truchement de Bertolt Brecht, nous pouvons constater qu'une photographie - qu'elle soit sociologique ou non - relève toujours d'une fabrication, d'une construction :

«La situation, dit Brecht, se complique du fait que, moins que jamais, une simple "reproduction de la réalité" n'explique quoi que ce soit de la réalité. Une photographie des usines Krupp ou AEG n'apporte à peu près rien sur ces institutions. La véritable réalité est revenue à la dimension fonctionnelle. La réification des rapports humains, c'est-à-dire par exemple l'usine elle-même, ne les représente plus. Il y a donc bel et bien "quelque chose à construire", quelque chose d'“artificiel", de "fabriqué". » (Benjamin, 1996, 10)

Et quelle que soit la boîte à outil méthodologique employée et le nombre de « photographies » réalisées par le sociologue, les représentations qu'il donne du terrain 
et de ceux qui y vivent ne sauraient recouvrir l'ensemble de ses réalités. Un écart reste présent, de la même façon qu'Hakim Bey souligne que la carte géographique est « une abstraction, elle ne peut pas couvrir la terre à l'échelle $1: 1$. Des complexités fractales de la géographie réelle, elle ne perçoit que des grilles dimensionnelles. Les immensités cachées dans ses replis échappent à l'arpenteur. La carte n'est pas exacte ; la carte ne peut pas être exacte. » (Bey, 1997, 17)

Il reste que ce qui apparaît aujourd'hui au sociologue comme plausible ne le sera plus forcément demain. En effet, rendre compte de son terrain d'enquête - en fabriquer un récit ou une représentation -, c'est décrire ici et maintenant une situation là-bas et déjà passée. Un ici et maintenant " grâce à quoi la réalité a pour ainsi dire brûlé de part en part. » (Benjamin, 1996, 10-11) C'est sans doute pourquoi les sociologues, cherchant à dégager un sens scientifique - une illusion de neutralisation (Mauger, op.cit., 128) - à leurs représentations, s'affairent à démultiplier les méthodes de prélèvement des données: approche ethnographique, enquête statistique, observation "directe» (participante ou non participante), monographie de terrain, entretiens «semi » ou « non directif », questionnaire en direction d'un échantillon dit "représentatif», etc. Ainsi les sociologues useront-ils alors - pour mesurer par exemple un différentiel dans le temps - d'une comparaison temporelle - qui est monnaie courante de l'engagement sociologique - pour tenter de vérifier, à partir d'une nouvelle photographie sociologique, les différences et similitudes d'un terrain déjà étudié.

51 Toutefois, lorsque le sociologue décrit et raconte ses terrains d'enquête, il révèle des photographies sociologiques qui relèvent d'observations de « faits » construites à partir de ses propres points de vue, de ses propres théories. Et si l'on peut dire avec Howard Becker qu' «il n'existe pas de faits purs, mais seulement des "faits" qui dérivent leur sens d'une théorie sous-jacente" (Becker, 2009, 26), décrire et raconter son terrain d'enquête - ou en faire la représentation - suppose alors une "trahison " - plus ou moins déformante - du terrain étudié.

Dans ces conditions, la représentation des mondes du travail serait-elle intimement liée au fait d'un « savoir-trahir »?

Pierre Bourdieu explique: "Très souvent en ethnologie, on a ce problème-là, les informateurs comme on les appelle, sont souvent des gens qui sont à la fois dedans et dehors, des gens qui sont marginaux dans le groupe, et de ce fait à la fois plus lucides et plus libres que les autres. Et ça, il faut des gens qui sont un peu traîtres, qui ont un peu mauvais esprit. Alors ça, ça fait gagner beaucoup de temps. ${ }^{30}$ "

En effet, qu'il fasse appel à un informateur - qui est à la fois dedans et dehors - ou qu'il sécrète ses propres sources (Mauger, op.cit., 125) - de l'intérieur -, le travail de l'enquêteur qui dévoile des "faits" étudiés sur son terrain requiert une certaine distance (un travail d'objectivation ou de "montée en généralité ») et de ce fait la capacité de savoir "trahir », y compris l'hospitalité de ses hôtes. Et l'on peut observer avec Michel de Certeau « une coupure entre le temps des solidarités (celui de la docilité et de la gratitude de l'enquêteur envers ses hôtes) et le temps de la rédaction qui dévoilent les alliances institutionnelles (scientifiques, sociales) et le profit (intellectuel, professionnel, financier, etc.) dont cette hospitalité est objectivement le moyen.» (de Certeau, 1990, 44)

Ce qui est valable pour le sociologue dans sa situation d'enquête l'est aussi pour l'artiste lorsqu'il est en position de représenter. L'acte de représentation des travailleurs 
implique effectivement une relation entre l'artiste et le travailleur qui n'apparaît que trop souvent hors champ de la représentation exposée au public-lecteur. On s'intéresse alors plus au résultat qu'au processus dont il est issu. Pourtant, on a pu constater que les méthodes employées pour représenter les travailleurs déterminent pour une grande part le positionnement et l'engagement de l'artiste vis-à-vis de ses modèles. L'acte de création constitue l'œuvre. Celle-ci est indissociable des conditions de sa production, qu'elles soient perceptibles ou non aux regards. Ainsi, les méthodologies engagées par les artistes dans l'acte de représentation et de monstration des travailleurs sont tout aussi politiques que les œuvres qui en résultent. Les liens d'interdépendance entre représentants et représentés nous obligent à reconsidérer la place de la subjectivité de l'auteur mais aussi celle de ses modèles. Les travailleurs représentés - comme les enquêtés - observent tout autant leurs représentants, qu'ils sont observés par eux. Pour reprendre l'expression de Gérard Mauger, la situation d'enquête - comme celle de l'acte de représentation - induit bien une situation d'examen réciproque (Mauger, 1992, 130). Même si cette situation d'examen entre l'enquêteur et l'enquêté est souvent asymétrique, l'enquêteur est enquêté par ses enquêtés comme le représentant est représenté par ses représentés.

Et l'expérience des groupes Medvedkine nous montre que cette subjectivité peut être mise en partage, transfigurée à la fois collectivement, poétiquement et politiquement. «Il faut faire politiquement des films politiques!» disait Jean-Luc Godard. Nous reprenons donc cette affirmation sous la forme d'une question: Comment les artistes font-ils pour représenter politiquement les travailleurs?

Représenter politiquement les travailleurs ouvriers, c'est tenter de les placer en position de pouvoir parler, de pouvoir s'auto-représenter. En effet, les travailleurs ont, pour reprendre l'expression de Pierre Bourdieu, des aptitudes inégales à prendre la parole dans les espaces de représentation publique et dans ces conditions: «Si l'on veut être égal, il faut être inégal, il faut soutenir ${ }^{31}$.» Soutien qui consistait, dans le cadre des groupes Medvedkine, à offrir aux travailleurs une situation de prise de parole. Pour faire en sorte qu'in fine ce soit les travailleurs eux-mêmes qui s'autoreprésentent. Pour les cinéastes des groupes Medvedkine, représenter politiquement les travailleurs, c'est donc d'abord tenter d'abolir les frontières entre représentants et représentés en contribuant à la rencontre des points de vue. L'apport méthodologique de ces groupes, c'est précisément ces tentatives d'inverser les rôles et les tâches attribués à chacun, y compris pour les techniciens du cinéma : «C'est assez émouvant de prendre le micro quand on en a pas l'habitude ", dit l'ingénieur du son Michel Desrois dans sa lettre à son ami Pol Cèbe ${ }^{32}$.

Aidés par le fait que les ouvriers ne se reconnaissaient pas dans les représentations qui étaient faites d'eux, les techniciens du cinéma ont renoncé à leur subjectivité unique et ont mis leur savoir-faire cinématographique au service d'une subjectivité collective, ou tout du moins partagée. De cette façon, ils ont placé les travailleurs de la Rhodiaceta en situation et en capacité de s'auto-représenter et de pouvoir répondre ainsi par leurs propres visions. Le film La charnière nous a permis de comprendre que le processus de représentation peut fonctionner à double sens. Il fallait que les ouvriers grévistes de la Rhodiaceta ne se sentent pas représentés par le film À bientôt j'espère... réalisé par Chris Marker et Mario Marret pour créer les conditions d'une prise en charge collective des films qui allaient désormais tenter de les représenter. 

l'engagement qui sous-tend sa démarche, savoir quelle place il prend ou laisse aux représentés en est une autre. Dans ces conditions, il apparaît que l'engagement de l'artiste vis-à-vis de son terrain et ses habitants varie en fonction de la place qu'il occupe et de la place qu'il laisse occuper - à toutes les étapes de la représentation - « à ceux qui sont habituellement les objets du discours des experts, à ceux dont on parle et qui restent silencieux sur leur propre expérience. » (Bourcier, 2001, 184)

61 Son engagement - s'il y a - fait qu'il ne révèle pas ses données de la même façon selon qu'il «baisse les yeux ou qu'il lève les yeux ", selon que les enquêtés occupent dans l'espace social une position dominante ou subalterne. Comme le dit Pierre Bourdieu : « Les gens ont très inégalement des choses à cacher. Les gens qui vous disent : "ça, vous n'enregistrez pas !", c'est quand même surtout les gens de pouvoir. Vous savez si vous parlez avec un chômeur ou un sous-prolétaire, qu'est ce qu'il a à cacher ${ }^{33}$ ? " alors que l'artiste se trouve toujours exposé à trahir son terrain et ses habitants. Cependant, «trahir », par exemple en révélant de l'intérieur du monde ouvrier - par le biais de la publication d'un journal de terrain et sur le mode de la dérision, de l'ironie et du gag - les transformations des conditions de travail - via l'accélération des cadences qui ont cours dans l'industrie automobile comme Hubert Truxler (alias Marcel Durand) (Durand, 2006) le fait, n'est pas la même chose que «trahir » en révélant d'une façon déformée le portrait photographique d'un ouvrier de chantier sans que celui-ci ait été tenu informé des intentions du photographe. A fortiori si cet ouvrier n'est pas en mesure de répondre à un miroir déformant qui reflète une image dans laquelle il ne se reconnaît pas ou dans laquelle il ne peut se reconnaître complètement.

63 Monter et rapporter des données prélevées sur son terrain d'enquête suppose alors une "trahison», plus ou moins acceptable, du terrain étudié et de ses habitants. Et il semble que s'engager veut dire pour les artistes - comme pour les sociologues qui, selon l'expression de Pierre Bourdieu, sont ces « troubles fête qui viennent détruire les communions magiques. » (Bourdieu, 1984, 197) - un « savoir-trahir » tout autant qu'un «savoir-faire » pour que les parlés parlent (Bourcier, 2001, 141).

\section{Conclusion}

Représenter les mondes du travail se révèle difficile et les modes de représentation qui intègrent cette difficulté et la mettent en scène sont probablement les plus aptes à échapper à l'écueil d'une simple illustration pour tenter de rendre compte du monde du travail dans sa complexité, tel qu'il est vécu par ses acteurs. L'artiste qui veut aujourd'hui donner à voir le monde du travail ne peut faire l'impasse sur cette réflexion quant à sa position.

65 L'artiste Philip-Lorca diCorcia paye ses modèles le prix d'une passe et expose dans la légende ce coût. Procédure méthodologique comparable à la rémunération qu'un enquêteur peut proposer à un enquêté afin de vaincre ses résistances et parvenir à obtenir de lui un entretien : «Il nous est arrivé également de rémunérer des entretiens [...] Le moyen s'est avéré efficace pour vaincre "les résistances" de certains jeunes de milieux populaires particulièrement "rétifs à l'enquête".» (Mauger, 1991, 132) Comme

Images du travail, travail des images, 2 | 2016 
l'artiste Philip-Lorca diCorcia, le sociologue Gérard Mauger assume la rémunération négociée avec ses enquêtés et l'intègre dans son compte rendu d'enquête de terrain.

Et si l'on ne peut augurer des intentions de l'artiste de participer généreusement à la réparation d'une invisibilité des sujets subalternes, Nicolas Faure, quant à lui, à partir d'une position d'artiste-invité, ne coopère avec ses modèles que faiblement et uniquement au moment de la prise de vue. Le processus qu'il a engagé nous apparaît prédéterminé par sa seule subjectivité d'auteur : il doit obtenir une photographie des ouvriers du chantier, afin que celle-ci vienne alimenter une série déjà engagée en dehors d'eux. Rien ne semble pouvoir venir bouleverser ce processus. Les ouvriers défilent devant l'appareil les uns après les autres et chaque nouveau cliché vient chasser le cliché précédent... Dans la représentation qu'il fait des ouvriers du chantier, il ne leur laisse aucune place pour échapper à une identité ouvrière prédéfinie.

Le travail de représentation artistique nous oblige à reconsidérer des procédures qui nous semblent d'ordinaire comme allant de soi. L'artiste comme le sociologue, lorsqu'ils représentent les travailleurs, ont tous deux intérêt à penser la représentation pour ne pas se substituer aux travailleurs en relayant des considérations préconçues (c'est-àdire parler ailleurs en leur nom et en devenir les experts ou les interprètes dans d'autres mondes). La position des travailleurs et la relation que l'on entretient avec eux doit être pensée et intégrée au processus de représentation.

Et quand bien même on peut dire avec Howard Becker que « toute représentation de la réalité sociale - film documentaire, étude démographique, roman réaliste - est nécessairement partielle, [car] elle contient moins que ce que l'on expérimenterait ou qui serait disponible à l'interprétation si l'on se trouvait dans le cadre réel qu'elle représente " (Becker, 2009, 34) et avec Jean-Claude Moineau qu'il y a toujours «non seulement de l'irréprétenté, des irreprésentés, de l'irreprésentable (voire de l'imprésentable); [et que de ce fait] on ne saurait jamais représenter l'entreprise en tnt que telle» (Moineau, 2015, 38), le possible de l'artiste - comme celui du sociologue pris dans une telle situation - et en dehors de toute définition des rôles impartis -, n'est-il pas justement de tenter de rétablir méthodologiquement les liens que le document révélé distend entre la «réalité expérimentée » et la représentation qu'il fabrique?

69 À défaut, les risques pour l'artiste d'être considéré par ses modèles comme une personne déloyale a priori (réputation de l'artiste) ou a posteriori (dans la restitution qu'il fait de ses données prélevées) resteront les lieux communs de l'expérience de l'artiste vis-à-vis de son terrain et de ses habitants.

70 Aussi, le recours au document produit - ou prélevé - de l'intérieur et par les intéressés eux-mêmes nous paraît essentiel car, en plus de construire une mémoire (celle des conditions de travail, des luttes et résistances...), il permet de contrecarrer l'image extérieure qu'entend donner d'elle l'entreprise et de produire ainsi une contre-culture, une culture parallèle critique. Témoigner des conditions de travail et/ou de résistance par des formes d'auto-représentation, c'est essayer de donner une autre version des mondes du travail, de montrer ce qui se cache derrière l'image policée par les services de communication - interne comme externe - à la solde des directions d'entreprises (sites Internet, publicités, enseignes...). Une autre vision de "l'intérieur » peut permettre ainsi de comprendre différemment l'organisation contemporaine de la production capitaliste et les nouvelles formes d'exploitation qu'elle impose aux 
travailleurs. Mais aussi les nouvelles formes de résistance qui lui font face. On ne peut représenter sans prendre part, et donc sans prendre parti.

De même que l'auto-représentation des conditions de vie en prison permet aux détenus de faire savoir à l'extérieur ce qui se passe à l'intérieur ${ }^{34}$, les formes d'autoreprésentation au travail sont indissociablement liées à des besoins de transformation par l'action coordonnée de ces mondes. Le caractère clandestin - ou semi-clandestin que suppose l'auto-représentation de ces mondes, et en l'absence de toutes autorisations, en fait également un acte performatif. En ce sens, il nous semble que les formes d'auto-représentation ne s'opposent pas aux formes d'actions directes de même que « la propagande par les mots, l'image... » ne s'oppose pas à « la propagande par les faits ». Tout comme les médiactivistes d'Indymedia par exemple, ne se contentent pas de pratiquer l'open publishing - «remettant ainsi en cause toute distinction entre auteurs et récepteurs de l'information en donnant à tous la possibilité de fournir le contenu rédactionnel et de partager les informations dont ils disposent " (Moineau, 2015, 15-16) - tout simplement parce que les médiactivistes participent également aux actions qu'ils relaient, et relaient les actions mêmes auxquelles ils participent. Si bien qu'action et (auto)représentation ne s'opposent pas, elles s'entrecroisent et se nourrissent mutuellement dans la nécessité de ne pas se contenter d'agir performativement sur le monde, mais d'agir de concert, ce qui suppose de s'engager aussi dans des formes d'(auto)représentation.

\section{BIBLIOGRAPHIE}

\section{Livres}

Bourcier M-H. (2001), Queer zones. Politique des identités sexuelles, des représentations et des savoirs, Paris, Balland.

Becker H. S. (2009), Comment parler de la société, Artistes, écrivains, chercheurs et représentations sociales, Paris, La Découverte.

Bey H. (1997), TAZ Zone Autonome Temporaire, Paris, l'Éclat.

Bourdieu P. (1984), Question de sociologie, Paris, Minuit.

Corouge C. et Pialloux M. (2001), Résister à la chaine, Dialogue entre un ouvrier de Peugeot et un sociologue, Marseille, Agone.

de Certeau M. (1990), L'Invention du quotidien tome 1 : arts de faire, Paris, Gallimard.

Durand M. (2006), Grain de sable sous le capot. Résistance et contre-culture ouvrière : les chaînes de montage de Peugeot (1972-2003), Marseille, Agone.

Marissal L. (2005), Pinxit, Rennes, Incertain Sens.

Marx K. (1969), Le capital, livre I, Paris, Garnier-Flammarion, 1969. 
Marx K. et Engels F. (1976), L'idéologie allemande, Paris, Ed. Sociales.

Moineau J-C. (2015), Artist as enterprise, Paris, Art Book Magazine, $2^{\text {ème }}$ Ed. Nicolas Ledoux.

Rosière S. (2010), Ouvrière d'usine ! Petits bruits d'un quotidien prolétaire, Ed. Libertaire.

Spivak G. (2009), Les Subalternes peuvent-elles parler ?, Paris, Amsterdam.

\section{Articles de revues}

Walter B. (1996), «Petite histoire de la photographie », Études photographiques, [En ligne], 1-1996, mis en ligne le 18 novembre 2002, consulté le 14 mars 2016. URL : http://

etudesphotographiques.revues.org/99

Chottin A. (2001), « L'échappée belle, entretien avec Christian Bernard », Vacarme, nº 14.

Nicolas-Le Start P. (1989), « D'une forme à l'autre, le salarié mis en scène », Les Temps Modernes, $n^{\circ} 514$.

Mauger G. (1991), « Enquêter en milieu populaire », Genèses, nº 6.

Middelbos J. (2011-2012), « Y a-t-il un artiste dans l'usine? », Art21, n 32.

Moineau J-C. (2008), « L'artiste et ses “modèles” ", NOIR, nº 1.

Molénat X. (2010), « Menaces sur le droit d'enquêter. Entretien avec Sylvain Laurens », Sciences Humaines, $\mathrm{n}^{\circ} 211$.

Muel B. (2000), «Les riches heures du groupe Medvedkine (Besançon-Sochaux, 1967-1974) », Images documentaires, $\mathrm{n}^{\circ} 37 / 38$.

Rouillé A. (1984), «Les images photographiques du monde du travail sous le second empire », le savoir-voir, Actes de la recherche en sciences sociales, $\mathrm{n}^{\circ} 54$.

Schönwald C. (2008), « Les Ateliers de Rennes, La fabrique de l'entente », Art 21, n 18.

\section{Catalogues}

Depardon R. (2000), Errance, Paris, Seuil.

Faure N., Lacoue-Labarthe P. et Nancy J-L. (2004), Portraits/Chantiers, Genève, Mamco.

Simpson B. (2007), Philip-Lorca diCorcia, Boston, The institute of contemporary art.

\section{Sites web}

Krieger M. «Chronologie de la réalisation du projet de tramway », L'art dans l'espace public, La commande publique à travers l'exemple de Strasbourg [En ligne], consulté le mercredi 10 mars 2010. URL : http://www.vision-unit.fr/strasbourg/principal.htm.

Krieger M. « Délibération du conseil municipal de la Communauté Urbaine de Strasbourg, le 8 octobre 1999 », Accompagnement artistique de la ligne "B" du tramway [En ligne], consulté le mardi 9 mars 2010. URL : http://www.vision-unit.fr/strasbourg/strasetapes/page01.htm\#04.

Lapp H. « Délibération du conseil municipal de la Communauté Urbaine de Strasbourg, le 8 octobre 1999 ", Accompagnement artistique de la ligne "B" du tramway, [En ligne], consulté le mardi 9 mars 2010. URL : http://www.vision-unit.fr. 
Le Faurestier A. Du travail de l'Art, à l'Art au travail..., [En ligne], site de l'artiste. URL : http:// aurelielefaurestier.blogspot.fr

\section{Films}

Anonyme, La prison de Fleury-Mérogis filmée clandestinement par des détenus , [En ligne], mis en ligne le 18 décembre 2008 et disponible sur le site du journal Le Monde, consulté le 7 mars 2010. URL : http://www.lemonde.fr

Bonfanti A. et Cèbe P. (1968), La Charnière, Éditions Montparnasse.

Carles P. (2002), Enfin pris, C-P Productions.

Marissal L. Sieste clandestine, [En ligne], site personnel de l'artiste. URL : http://painterman.overblog.com

Desrois M. (Groupe Medvedkine) (1970), Lettre à mon ami Pol Cèbe, Ed. Montparnasse.

\section{NOTES}

1. La polysémie du terme de « représentation ", l'écran de quiproquos qu'il induit toujours, nous oblige à différencier ici - et au-delà de son sens étymologique d' " action de replacer devant les yeux de quelqu'un » - le moment de la représentation "politique » (la Vertretung en allemand) qui implique la délégation d'un pouvoir d'agir à un mandataire, d'une proposition à fonction de représentation "figurative" (la Darstellung en allemand) que nous utiliserons ici au sens de son expression "symbolique", comme l'action de rendre quelque chose, quelqu'un ou un groupe social présent, visible - aussi lointain soit-il - sous la forme d'un substitut ou en recourant à un artifice.

2. Présentation du colloque organisé par le Gresco, la MSHS et l'université de Poitiers du 8 au 17 février 2013, «Image du travail, travail des images. Pratiques artistiques, démarches scientifiques » [En ligne] consulté le lundi16 juillet 2015. URL : http://2013.filmerletravail.org.

3. Ce qu'on appelle également en termes sociologiques faire de «l'observation à couvert » ou encore de «l'observation clandestine ».

4. À « supposer même que dans certaines conditions sociales chaque individu soit un excellent peintre, cela n'exclurait en aucune façon que chacun fût un peintre original, si bien que, là aussi, la distinction entre travail « humain » et travail « unique » aboutisse à un pur non-sens. Dans une organisation communiste de la société, ce qui sera supprimé en tout état de cause, ce sont les barrières locales et nationales, produits de la division du travail, dans lesquelles l'artiste est enfermé, tandis que l'individu ne sera plus enfermé dans les limites d'un art déterminé, limites qui font qu'il y a des peintres, des sculpteurs, etc., qui ne sont que cela, et le nom à lui seul exprime suffisamment la limitation des possibilités d'activité de cet individu et sa dépendance par rapport à la division du travail. Dans une société communiste, il n'y aura plus de peintres, mais tout au plus des gens qui, entre autres choses, feront de la peinture. » (Marx et Engels, 1976, 397).

5. «La puissance sociale, c'est-à-dire la force productive décuplée qui naît de la coopération des divers individus conditionnés par la division du travail, n'apparaît pas à ces individus comme leur propre puissance conjuguée, parce que cette coopération elle-même n'est pas volontaire mais naturelle ; elle leur apparaît au contraire comme une puissance étrangère, située en dehors d'eux dont ils ne savent ni d'où elle vient, ni où elle va, qu'ils ne peuvent donc plus dominer et qui, à l'inverse, parcourt maintenant une série particulière de phases et de stades de 
développement, si indépendante de la volonté, qu'elle dirige en vérité cette volonté et cette marche de l'humanité. » (Marx, 1969, 266-267).

6. Aurélie Le Faurestier, Du travail de l'Art, à l'Art au travail..., site de l'artiste: http:// aurelielefaurestier.blogspot.fr/.

7. Michel Krieger était, de 1991 à 2001, président du groupe d'experts, conseiller municipal à la ville de Strasbourg, conseiller à la Communauté urbaine de Strasbourg, et délégué à la commande publique et à la mise en place de l'art dans l'espace public.

8. Christian Bernard est fondateur du Mamco (Musée d'art moderne et contemporain de Genève).

9. C'est le conseil de la Communauté urbaine de Strasbourg qui a approuvé, lors de sa séance du 15 décembre 1995, le principe d'un accompagnement artistique de la ligne "B" du tramway. Cette séance (6 novembre 1998) a confirmé la réalisation de ce projet pour un montant total de 8 Millions de Francs, financé paritairement par l'État via le ministère de la Culture (4 MF), et par la Communauté urbaine de Strasbourg (4 MF).

Krieger M. "Chronologie de la réalisation du projet de tramway ", L'art dans l'espace public. La commande publique à travers l'exemple de Strasbourg [En ligne], consulté le mercredi 10 mars 2010. URL : http://www.vision-unit.fr/strasbourg/principal.htm ; et Chottin A. (2001), «L'échappée belle, entretien avec Christian Bernard », Vacarme, n¹4, p. 107-110.

10. En 1992, un "accompagnement artistique » de l'implantation de la première ligne du tramway (ligne A) avait déjà été établi sur le principe d'un financement paritaire entre la ville de Strasbourg et le ministère de la Culture (pour une enveloppe totale de six millions de francs). C'est Jean Christophe Ammann qui était le chef du projet artistique de la ligne A. (Krieger, Ibid).

11. Sept artistes dont un photographe et une architecte-plasticienne (Siah Armajani, Bert Theis, Jean-Luc Vilmouth, Alain Séchas, Jean-Marie Krauth, Zaha Hadid, Nicolas Faure), un musicien (Rodolphe Burger), huit écrivains de divers genres (Pierre Ahnne, Didier Daeninckx, Michel Deutsch, Jacques Roubaud...), le laboratoire de tourisme expérimental de Joël Henry ainsi que deux philosophes (Jean-Luc Nancy et Philippe Lacoue-Labarthe), ont été associés à cette commande autour de la ligne B du tramway. (Chottin, op.cit).

12. Krieger M. « Délibération du conseil municipal de la Communauté urbaine de Strasbourg, le 8 octobre 1999 ", Accompagnement artistique de la ligne "B" du tramway [En ligne], consulté le mardi 9 mars 2010. URL : http://www.vision-unit.fr/strasbourg/strasetapes/page01.htm\#04

13. Ibid.

14. «Comme nous l'avons fait pour la ligne A, avec le succès qu'a rencontré cette commande publique qui, malgré les défauts que l'on a pu constater sur l'une des œuvres, a néanmoins accru la notoriété de Strasbourg et a été appréciée, à bien des égards, en France et à l'étranger, comme une démarche exemplaire. Certes, des noms comme Zaha Hadid, Siah Armajani, comme ceux d'Alain Sechas, de Vilmouth, de Krauth ou de Nicolas Faure, ne manqueront pas d'attirer l'attention de la presse et des experts des milieux culturels européens sur ce qui se fait à Strasbourg. L'intervention de Bert Theiss mettra Strasbourg au rang des métropoles qui comptent dans l'art contemporain en Europe. » Michel (Krieger, op.cit.).

15. C'est le conseiller municipal strasbourgeois, Michel Krieger, qui est à l'origine et qui préside ce projet d' " accompagnement artistique » de la construction des tramways, sous l'autorité de Roland Ries, maire (PS) et président de la Communauté urbaine de Strasbourg de 1997 à 2000.

16. Harry L. «Délibération du conseil municipal de la Communauté Urbaine de Strasbourg, le 8 octobre 1999 ", Accompagnement artistique de la ligne "B" du tramway [En ligne], consulté le mardi 9 mars 2010. URL : http://www.vision-unit.fr

17. "Je parlerais de remerciement, si le mot ne rappelait justement pas, en latin, le salaire.» Merci, provient du latin mercedem, de merces " salaire, récompense, solde, intérêt, rapport ». Trésor de la langue française, dictionnaire de la langue du $19 \mathrm{e}$ et du 20e siècle, dit TLF, édité par le CNRS et les Éditions Gallimard [En ligne], consulté sur le le mardi 30 mars 2010. URL : http:// atilf.atilf.fr/tlf.htm 
18. Nous avons également cherché à contacter Stéphane Fond - malheureusement sans succès pour l'avertir de la publication future de cet article et de cette photographie dans la revue en ligne Images du travail, travail des images.

19. Entretien téléphonique réalisé avec Stéphane Fond le lundi 15 mars 2010.

20. Ibid.

21. Nous distinguons ici le moment de la représentation «politique » (la Vertretung en allemand) qui implique la délégation d'un pouvoir d'agir à un mandataire, d'une proposition à fonction de représentation «figurative" (la Darstellung en allemand) que nous utiliserons ici au sens de son expression « symbolique ». Voir note $n^{\circ} 1$.

22. J'ai posé la question à Stéphane Fond - qui a servi de modèle pour le photographe ? - afin de savoir si tous ses collègues avaient également accepté d'être photographiés. Voici sa réponse : «Non, les anciens ont refusé de faire la photographie. On était juste deux ou trois à poser.» Entretien téléphonique réalisé le lundi 15 mars 2010.

23. Raymond Depardon, en se situant quelque part à la frontière entre la photographie de paysage et de portrait, revendique sa propre distance - qu'il qualifie d'éthique - par rapport au sujet qu'il photographie: "Il y a une espèce d'éthique à ne pas photographier de trop près. » (Depardon, 2000, 32).

24. «Ceux qui d'ordinaire se laissaient docilement interviewer, questionner, objectiver par les sociologues n'hésitent désormais plus à contrôler l'usage qui est fait des données qui les concernent. " Molénat X. (2010), "Menaces sur le droit d'enquêter, Entretien avec Sylvain Laurens", Sciences Humaines, Le clash des idées - 20 livres qui ont changé notre vision du monde, Mensuel $n^{\circ} 211$.

25. En témoigne cette confession de Bruno Muel qui doute de son rôle et des rôles de chacun dans le processus de fabrication du film Week-end à Sochaux tourné en 1971 : «Mon plus gros problème était de filmer les scènes de fiction. Je me disais: "Mais qu'est-ce que tu vas faire là. Qui est le réalisateur? C'est toi ou c'est eux ?" Il faut remarquer que la première utopie des groupes Medvedkine était passée à la trappe. Ils se foutaient complètement de la technique, tenir la caméra ne les intéressaient pas du tout, nous étions là pour ça. Eux, ce qu'ils voulaient c'étaient faire passer leurs idées, leur point de vue. » (Muel, 2000, 29-30).

26. Les jeunes ouvriers des usines Peugeot «n'hésitaient pas à faire le trajet jusqu'à Clermoulin [base arrière des groupes Medvedkine] et à consacrer leur temps à concocter des films après leur journée de chaîne à l'usine ou pendant les week-ends. [...] Ils faisaient le trajet presque tous les soirs, ça dépendait de quelle tournée ils étaient, et tous les week-ends. D’où le titre du film Weekend à Sochaux. » (Muel, ibid., 27-29).

27. Les processus de "délocalisation » et de "relocalisation » des centres vers les périphéries actuellement en cours, dans le cadre de la division internationale du travail, permettent aussi aux dirigeants d'entreprise de cacher les conditions de production. Ce qui revient à tenter de briser encore un peu plus les liens d'interdépendance et de solidarité (ce que Durkheim nomme les «solidarités organiques») qui peuvent exister entre les producteurs et les «usagersconsommateurs ».

28. Cf. Schönwald C. (2008), «Les Ateliers de Rennes, La fabrique de l'entente », art 21, n¹8, p. 32-39.

29. Laurent Marissal, Sieste clandestine, site personnel de l'artiste: http://painterman.overblog.com.

30. Pierre Bourdieu, dans le film Enfin pris de Pierre Carles, C-P Productions, 2002.

31. Pierre Bourdieu, ibid.

32. Michel Desrois dans son film court métrage Lettre à mon ami Pol Cèbe, Éditions Montparnasse, 1970.

33. Pierre Bourdieu, $i b i d$. 
34. On pense ici aux films réalisés - clandestinement et avec des caméras introduites illégalement dans la prison de Fleury-Mérogis - par les détenus eux-mêmes, afin de témoigner et de dénoncer leurs conditions de vie carcérale. Le Monde, « La prison de Fleury-Mérogis filmée clandestinement par des détenus » [En ligne], en ligne le 18 décembre 2008, consulté le 7 mars 2010. URL : http:// www.lemonde.fr

\section{RÉSUMÉS}

Cet article fait retour sur une communication prononcée dans l'atelier intitulé L'artiste au travail lors du colloque Images du travail, Travail des images (Poitiers, le 13 février 2013). Il s'agissait de discuter - entre autres - des méthodes de représentation artistique des mondes du travail en interrogeant plus particulièrement la position de l'artiste en rapport avec ses modèles, les travailleurs. Comment l'artiste accède-t-il à son terrain, à son décor ? Est-il lui-même salarié de l'entreprise ou est-il en visite dans l'entreprise ? Et s'il est un artiste invité dans l'entreprise, comment négociera-t-il sa mise en scène avec les travailleurs? Dans quel contexte et pour quel public exposera-t-il ces représentations? Et pour quel profit (intellectuel, professionnel, financier, etc.)?

Autant de questions qui n'apparaissent le plus souvent qu'hors champs de la représentation mais qui, lorsqu'on s'y intéresse, n'en constituent pas moins des indices sur les conditions de production et de monstration des images du travail, sur les rapports contractuels que les artistes entretiennent avec les travailleurs, sur les déséquilibres subjectifs - produit de la division du travail entre représentants et représentés - qui persistent entre les artistes et les travailleurs...

À l'aide d'un corpus artistique que nous pouvons d'ores et déjà placer sous la catégorie esthétique de l'art documentaire, nous chercherons à remonter le fil méthodologique de ces représentations mises en place par l'artiste. Il s'agira donc de savoir comment l'artiste-invité, qui cherche à pallier à l'invisibilité des travailleurs, peut-il rétablir méthodologiquement ces déséquilibres subjectifs entre l'auteur et son modèle, mais aussi quel autre point de vue le salarié-artiste peut-il proposer de l'intérieur même des mondes du travail qu'il habite.

This article is the result of a presentation made in the workshop "The artist at work", in the colloquium Images of work, Work of Images (Poitiers, February 13, 2013). The purpose is to discuss the methods of artistic representation of the working worlds and to interrogate the position of the artist in relation with his models, the workers. How does the artist get into his field, his setting? Is he an employee or a visitor? If he's been invited to the enterprise, how will he negotiate the mise-en-scene with the workers? In what context and for what type of public will he show his final work? What would be the benefit (intellectual, professional, financial, etc.)?

We want to focus on questions that arise both inside and outside the field of artistic representation. Among them, the terms of production and display of the images, the contractual relationship that the artists have with the workers, the "imbalance subjective" (product of the division of labor between the representatives and the represented) that persist between the artists and the workers, etc.

We will follow methodologies used by artists who work in the aesthetic category of documentary art. The aim is to know how the invited-artist diminishes the invisibility of the workers, but also how he can restore the "imbalance subjective" between the author and his model, and what other perspective the employee-artist can propose from the world of work that he live in. 
INDEX

Mots-clés : représentation, travailleur, méthode, divisions du travail, artiste-invité, mise en scène, salarié-artiste

Keywords : representation, worker, method, division of labor, invited-artist, mise-en-scene, employee-artist

\section{AUTEUR}

JAN MIDDELBOS

Jan Middelbos est ouvrier du spectacle (sous le régime des intermittents du spectacle annexe 8) et doctorant en esthétique à l'université de Rennes 2. Il travaille sur les représentations artistiques du monde du travail à l'époque du travail dit « immatériel » et sur les pratiques tactico-déviantes au travail. 